\title{
Unraveling the temperature dependence of the yield strength in single-crystal tungsten using atomistically-informed crystal plasticity calculations
}

\author{
David Cereceda $^{\mathrm{a}, \mathrm{b}, \mathrm{c}}$, Martin Diehl ${ }^{\mathrm{d}}$, Franz Roters ${ }^{\mathrm{d}}$, Dierk Raabe ${ }^{\mathrm{d}}$, J. Manuel Perlado ${ }^{\mathrm{c}}$, Jaime Marian ${ }^{\mathrm{a}, *}$ \\ ${ }^{a}$ Department of Materials Science and Engineering, University of California Los Angeles, Los Angeles, CA 90095, USA \\ ${ }^{b}$ Physical and Life Sciences Directorate, Lawrence Livermore National Laboratory, Livermore, CA, USA \\ ${ }^{c}$ Instituto de Fusión Nuclear, Universidad Politécnica de Madrid, E-28006 Madrid, Spain

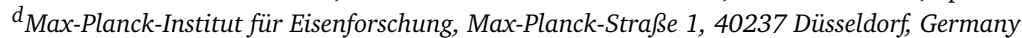

\begin{abstract}
We use a physically-based crystal plasticity model to predict the yield strength of body-centered cubic (bcc) tungsten single crystals subjected to uniaxial loading. Our model captures the thermally-activated character of screw dislocation motion and full non-Schmid effects, both of which are known to play a critical role in bcc plasticity. The model uses atomistic calculations as the sole source of constitutive information, with no parameter fitting of any kind to experimental data. Our results are in excellent agreement with experimental measurements of the yield stress as a function of temperature for a number of loading orientations. The validated methodology is then employed to calculate the temperature and strain-rate dependence of the yield strength for 231 crystallographic orientations within the standard stereographic triangle. We extract the strain-rate sensitivity of $\mathrm{W}$ crystals at different temperatures, and finish with the calculation of yield surfaces under biaxial loading conditions that can be used to define effective yield criteria for engineering design models.
\end{abstract}

Keywords: Bcc crystal plasticity, Yield stress, Non-Schmid effects, Screw dislocations, Single crystal tungsten, Uniaxial/biaxial loading

\section{Background and motivation}

The plastic behavior of body-centered cubic (bcc) single crystals at low to medium homologous temperatures is governed by the motion of $\frac{1}{2}\langle 111\rangle$ screw dislocations on close-packed crystallographic planes. There are two particularities that make bcc metals unique in relation to their deformation characteristics. The first one is the thermally-activated nature of screw dislocation glide, a consequence of the compact (non-planar) structure of the dislocation core at the atomistic level (Vitek, 2004; Wurster et al., 2010; Li et al., 2012; Samolyuk et al., 2013). This feature is also responsible for the high intrinsic friction stresses reported in the literature for bcc metals and their alloys (Romaner et al., 2010; Samolyuk et al., 2013). The second is the breakdown of the standard geometric projection rule for the resolved shear stress (RSS) from the total stress tensor known as Schmid law (Schmid \& Boas, 1935). This is owed to both specific crystallographic properties of the bcc lattice structure as well as to the coupling between the dislocation core and non-glide components of the stress tensor, which -to the best of our understanding-is unique to bcc crystals (Bulatov et al., 1999; Brinckmann et al., 2008; Woodward \& Rao, 2001; Chaussidon et al., 2006; Gröger \& Vitek, 2005). These anomalies have been the subject of much research and discussion going back to the 1960's

\footnotetext{
* Corresponding author

Email address: jmarian@ucla.edu (Jaime Marian)
}

Preprint submitted to International Journal of Plasticity

August 19, 2015

(C) 2015. This manuscript version is made available under the Elsevier user license http://www.elsevier.com/open-access/userlicense/1.0/ 
(Takeuchi et al., 1967; Hull et al., 1967; Duesbery, 1969; Duesbery \& Foxall, 1969), both experimentally and -more recently- using computational atomistic models.

In regards to the first point above, at low stresses slip proceeds via the thermally activated nucleation of steps on the dislocation line, known as kink pairs, and their subsequent sideward relaxation. For a constant strain rate, this gives rise to the characteristic temperature dependence of the flow stress in bcc single crystals, which has been observed for all refractory metals and is considered to be a principal signature of their plastic response (Seeger, 1981; Ackermann et al., 1983; Taylor, 1992; Gordon et al., 2010; Chaussidon et al., 2006; Yang et al., 2001). The flow stress is considered to be composed of thermal and athermal contributions, with the latter depending on temperature only as the elastic moduli. Dislocation glide is thought to occur on $\{110\},\{112\}$, and even $\{123\}$ planes, depending on temperature and stress, over a periodic energy landscape known as the Peierls potential $U_{P}$. The connection between the experimentally measured flow stress and this periodic energy potential is via the critical stress for which $U_{P}$ vanishes at zero temperature, known as the Peierls stress $\sigma_{P}$. Theoretically then, the flow stress at very low temperatures $(\leqslant 25 \mathrm{~K})$ is thought to represent the macroscopic equivalent of $\sigma_{P}$ as the temperature approaches $0 \mathrm{~K} . \sigma_{P}$ can thus be unequivocally defined and has been the object of considerable numerical work since the first atomistic models were devised by Vitek and co-workers starting in the 1970s (Vitek \& Yamaguchi, 1973).

For their part, non-Schmid effects were detected in tests done in the 1930's by Taylor in the wake of his seminal works on plastic flow and strain hardening (Taylor, 1928, 1934a,b). Subsequent observations and measurements (Šesták \& Zárubová, 1965; Sherwood et al., 1967; Zwiesele \& Diehl, 1979; Christian, 1983; Pichl, 2002; Escaig, 1968, 1974), and a rigorous theoretical formulation of the problem (Duesbery \& Vitek, 1998; Ito \& Vitek, 2001; Woodward \& Rao, 2001; Gröger \& Vitek, 2005; Chaussidon et al., 2006; Gröger et al., 2008a,b; Soare, 2014) have established non-Schmid behavior as a principal tenet of bcc plasticity that must be accounted for in order to understand bcc plastic flow. In terms of phenomenology, the two essential aspects to bear in mind are (i) that the resolved shear stress is not independent of the sign of the stress in glide planes of the $\langle 111\rangle$ zone (the so-called twinning/anti-twinning asymmetry), and (ii) that non-glide components of the stress tensor -i.e. those which are perpendicular to the Burgers vector-play a role on the magnitude and sign of the RSS on the glide plane of interest.

Areas where we do not have a complete understanding of bcc plastic picture include the value of the flow stress at near zero absolute temperatures, the meaning of the so-called knee temperature, and the onset of athermal flow. In the last two decades, computer simulation has unquestionably emerged as discipline capable of shedding light on these processes on a similar footing with experiments, providing physically-substantiated explanations across a range of temporal and spatial scales. These include the use and application of density-functional theory methods (Ventelon \& Willaime, 2007; Ventelon et al., 2013; Weinberger et al., 2013; Dezerald et al., 2014, 2015), semi empirical atomistic calculations and molecular dynamics calculations (Gilbert et al., 2011; Queyreau et al., 2011; Chang et al., 2001; Komanduri et al., 2001), kinetic Monte Carlo (Lin \& Chrzan, 1999; Cai et al., 2002; Deo \& Srolovitz, 2002; Scarle et al., 2004; Stukowski et al., 2015), and crystal plasticity (CP) (Qin \& Bassani, 1992; Dao \& Asaro, 1993; Brünig, 1997), to name but a few. In general, while there is no doubt that the intricacies associated with $\frac{1}{2}\langle 111\rangle$ screw dislocation glide -including its thermally activated nature and deviations from Schmid law- cannot but be resolved using methods capable of atomistic resolution, one must recognize that, at the same time, flow is a phenomenon potentially involving statistically-significant amounts of dislocations and -as such- cannot be captured resorting to atomistic calculations only.

Modeling thermally-activated flow and non-Schmid effects in bcc systems has been the subject of much work, starting in the 1980s and, particularly, in recent times. Different authors have considered different subsets of the $\{110\},\{112\}$, and $\{123\}$ families of glide planes, without (Raphanel \& Van Houtte, 1985; Hölscher et al., 1991, 1994; Raabe et al., 1994; Raabe, 1995a,b; Peeters et al., 2000; Stainier et al., 2002; Erieau \& Rey, 2004; Ma et al., 2007; Hamelin et al., 2011; Kitayama et al., 2013) and with non-Schmid effects (Lee et al., 1999; Kuchnicki et al., 2008; Koester et al., 2012; Weinberger et al., 2012; Alankar et al., 2014; Lim et al., 2013; Narayanan et al., 2014; Patra et al., 2014; Knezevic et al., 2014; Lim et al., 2015a,b). Of particular interest are some recent simulations where the flow rule is directly formulated on the basis of screw dislocation properties in Fe (Yalcinkaya et al., 2008; Koester et al., 2012; Alankar et al., 2014; Narayanan et al., 2014; Patra et al., 2014; Lim et al., 2015b), Ta (Kuchnicki et al., 2008; Lim et al., 2013; 
Knezevic et al., 2014; Lim et al., 2015a), Mo (Yalcinkaya et al., 2008; Weinberger et al., 2012; Lim et al., 2013, 2015a), W (Lee et al., 1999; Weinberger et al., 2012; Lim et al., 2013; Knezevic et al., 2014; Lim et al., 2015a), and Nb (Yalcinkaya et al., 2008; Lim et al., 2015a). These works also include non-Schmid effects following the model proposed by Vitek and Bassani (Duesbery \& Vitek, 1998; Qin \& Bassani, 1992; Gröger et al., 2008a,b). However, albeit very useful for certain applications, all these works resort to (i) a partial consideration of non-Schmid effects, and (ii) some kind or another of parameter fitting with experimental data, which prevents their use in regions of the parameter space outside the range of fitting and does not link the effective (macroscopic) response to exclusively fundamental material properties and features.

In this work, we provide a unified computational methodology consisting of rate-dependent crystal plasticity calculations parameterized entirely and exclusively to atomistic calculations. We show that a full description of non-Schmid effects, together with the state of the art in terms of our understanding of thermally-activated screw dislocation motion, suffices to capture the experimentally measured temperature dependence of the flow stress in tungsten. This is achieved in a fully classical framework, without the need for quantum effects recently invoked to explain the long standing discrepancy observed between the experimentally-measured flow stress below $25 \mathrm{~K}$ and calculated values of the Peierls stress (Proville et al., 2012). Our methodology also captures the athermal limit of $\mathrm{W}$ to within $5 \%$ of the experimental value. We emphasize that this agreement is reached without fitting to any experimental data, all the parameterization is done from first principles atomistic calculations.

Our paper is organized as follows. After this introduction, we provide an overview of the CP method in Section 2.2. This is followed by Sections 2.3.1 and 2.3.2, where the formulation of the dislocation mobility law and the implementation of non-Schmid effects are presented, including a detailed description of the parameterization procedure employed. The results are given in Section 3, which includes: (i) the validation exercise, with special focus on uniaxial tests as a function of temperature for several loading orientations; (ii) the calculation of temperature and strain rate dependence of the yield strength for uniaxial tensile tests as a function of orientation; and (iii) yield surfaces under biaxial loading conditions as a function of temperature. We finalize in Section 4 with a brief discussion and the conclusions.

\section{Computational methods}

\subsection{Flow kinematics}

Roters et al. (2010) have presented a detailed review of the kinematic and constitutive aspects of crystal plasticity and here we simply provide a brief overview of the fundamental theory. The kinematics for elastoplastic behavior is defined within the finite deformation framework. The material deformation involves both a reversible lattice response to externally imposed loads or displacements (elastic), and a permanent deformation (irreversible shape change) that remains after all external constraints cease to be applied (plastic). Consequently, crystal plasticity formulations rely on the definition of three reference systems: (i) a fixed coordinate system that represents a laboratory (undeformed) frame of reference, (ii) a current (also known as material) frame of reference that represents the global (deformed) shape of the material, and (iii) a lattice coordinate system that represents distortions of the underlying crystal structure of the deformed body. Although reference system (i) is used for mathematical convenience, the distinction between (ii) and (iii) is necessary to calculate internal stresses, which arise from distortions defined with respect to a crystallographic frame of reference, as global shape changes may not necessarily have a one-to-one correspondence to internal lattice distortions (Lubliner, 2008; Roters et al., 2010).

Mathematically, each point $\boldsymbol{X}$ in the reference configuration may be mapped to its image in the current configuration $\boldsymbol{x}$ by means of a linear transformation represented by the deformation gradient tensor $\boldsymbol{F}$, defined as:

$$
\boldsymbol{F}=\frac{\partial \boldsymbol{x}}{\partial \boldsymbol{X}}
$$

In general, $\boldsymbol{F}$ is not a symmetric tensor. However, invariance requirementsmake it more desirable to work with symmetric measures of strain. One such measure is the so-called Lagrangian strain:

$$
\boldsymbol{E}=\frac{1}{2}(\boldsymbol{C}-\boldsymbol{I})=\frac{1}{2}\left(\boldsymbol{F}^{T} \boldsymbol{F}-\boldsymbol{I}\right)
$$


which refers the deformation of the solid to the reference configuration ( $I$ is the identity tensor). In the above equation, $C$ is the so-called right Cauchy-Green tensor.

Following Lee (1969), the total deformation gradient $F$ can be multiplicatively decomposed into an elastic, $\boldsymbol{F}_{e}$, and a plastic, $\boldsymbol{F}_{p}$, part ${ }^{1}$, i.e.:

$$
\boldsymbol{F}=\boldsymbol{F}_{e} \boldsymbol{F}_{p}
$$

whence

$$
\boldsymbol{F}_{e}=\boldsymbol{F} \boldsymbol{F}_{p}^{-1} \Leftrightarrow \boldsymbol{F}_{p}=\boldsymbol{F}_{e}^{-1} \boldsymbol{F}
$$

This is schematically shown in Figure 1, where the relationship between the reference, intermediate, and current configurations is provided. To close the CP model, we take the time rate in eq. (1), which results in:

$$
\boldsymbol{L}=\dot{\boldsymbol{F}} \boldsymbol{F}^{-1}=\dot{\boldsymbol{F}}_{e} \boldsymbol{F}_{e}^{-1}+\boldsymbol{F}_{e}\left(\dot{\boldsymbol{F}}_{p} \boldsymbol{F}_{p}^{-1}\right) \boldsymbol{F}_{e}^{-1}=\boldsymbol{L}_{e}+\boldsymbol{F}_{e} \boldsymbol{L}_{p} \boldsymbol{F}_{e}^{-1}
$$

where $\boldsymbol{L}_{p}$ is the plastic velocity gradient, which is evaluated in the intermediate configuration and must therefore be mapped into the current configuration by $\boldsymbol{F}_{e}$. Constitutive information enters the CP model via $L_{p}$, which is described in the following section.

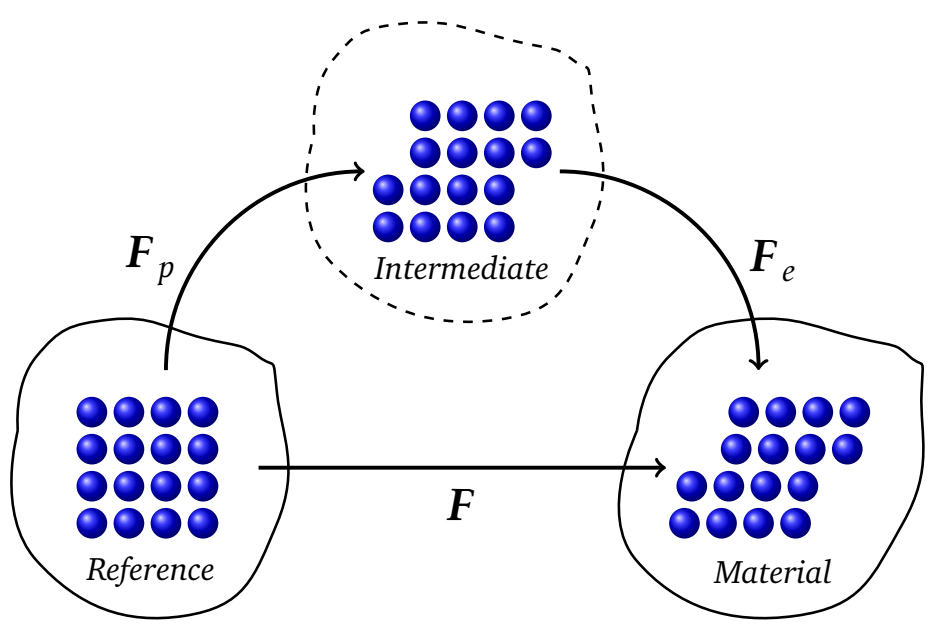

Figure 1: Multiplicative decomposition of the deformation gradient $\boldsymbol{F}$.

The above finite-deformation kinematic frameworkis implemented into the Düsseldorf Advanced Materials Simulation Kit (DAMASK), which is the tool employed in this work to carry out of the calculations. DAMASK is a flexible and hierarchically structured model of material point behavior for the solution of elastoplastic boundary value problems along with damage and thermal physics (Roters et al., 2012).

\subsection{Solution procedure and constitutive model}

A Hookean constitutive response is assumed such that the stress depends linearly on the elastic strain via the anisotropic elastic stiffness tensor $\mathbb{C}$. Both the stress and strain measures that are used internally are formulated in terms of material coordinates. For the stress, we use the second Piola-Kirchhoff stress measure $S$, defined as:

$$
S=\mathbb{C}: E_{e}=\frac{\mathbb{C}}{2}\left(F_{e}^{T} F_{e}-I\right)
$$

\footnotetext{
${ }^{1}$ It must be noted that other decompositions are also admissible (Fish \& Shek, 2000). The reader is referred to the work by Reina \& Conti (2014) for a discussion on the uniqueness and validity of the multiplicative decomposition.
} 
where $\boldsymbol{E}_{\boldsymbol{e}}$ is the (elastic) Green-Lagrange strain tensor. $S$ and $\boldsymbol{E}_{\boldsymbol{e}}$ are both symmetric material tensors, and thus $\mathbb{C}$ is itself symmetric such that a general $3 \times 3 \times 3 \times 3$ tensor can be written as a $6 \times 6$ matrix. For cubic lattices, $\mathbb{C}$ can be reduced by symmetry to only the three independent elastic constants $\mathbb{C}_{11}, \mathbb{C}_{12}$, and $\mathbb{C}_{44}$.

The stress $S$ acts as the driving force for the plastic velocity gradient $\boldsymbol{L}_{p} . \boldsymbol{L}_{p}$ depends on the underlying microstructure via a set of state variables $\xi$ defined by the plasticity model employed:

$$
L_{p}=f(S, \xi, \ldots)
$$

$\boldsymbol{L}_{p}$ controls the evolution of the plastic deformation gradient:

$$
\dot{\boldsymbol{F}}_{p}=\boldsymbol{L}_{p} \boldsymbol{F}_{p}
$$

The set of nonlinear eqs. (3) and (5) to (7) must be solved iteratively, which in DAMASK is done by using an integration algorithm based on the implicit scheme originally proposed by Kalidindi et al. (1992). The linear system is solved iteratively using the Newton-Raphson technique and, once convergence is achieved, the plastic deformation gradient is obtained using the Euler backward update. In this integration scheme described above, the primary variable to solve for is the plastic velocity gradient. However, one may devise schemes where the primary variables are the stress, the plastic or elastic deformation gradient, the internal variables or a combination thereof. Such schemes may be chosen on the basis of computational efficiency (Dumoulin et al., 2009).

Constitutive information for the plastic regime enters the CP model via eq. (6), where the dependencies of the flow rule on each of the state variables are established. It is here where the plastic deformation modes are defined, their geometric particularities, as well as specifics associated with the crystal structure under study. The CP model must also include evolution equations for the state variables $\xi$ :

$$
\dot{\xi}=g(S, \xi, \ldots)
$$

where the details again depend on the model selected. In DAMASK, various integration schemes for the state update exist (Roters et al., 2012). Then, two integration schemes are performed staggered: eqs. (3) to (7) are solved at a fixed plastic state, followed by a state update. This procedure is iteratively repeated until a converged solution is achieved within the given tolerances. More details about the implementation of this technique in the code are given by Kalidindi et al. (1992). In general then, the stress in the CP model can be considered a response function of the position $\boldsymbol{r}$, the deformation state $\boldsymbol{F}$, the set of state variables $\xi$, and a set of boundary conditions, i.e.

$$
\boldsymbol{S}=f(\boldsymbol{r}, \boldsymbol{F}, \boldsymbol{\xi}, \ldots)
$$

In these calculations we are interested in simulating engineering stress-strain tests, and -consequentlyit is helpful to express the results in the reference coordinate frame. For the stress, we use the first PiolaKirchhoff measure defined as:

$$
\boldsymbol{P}=\boldsymbol{F}_{e} S
$$

Note that, although in general $\boldsymbol{P}$ is not symmetric, for uniaxial tension tests $\boldsymbol{P}$ is a symmetric tensor on account of $\boldsymbol{F}_{e}$ being symmetric. For the strain, we use the Biot tensor:

$$
\boldsymbol{B}=\boldsymbol{U}-\boldsymbol{I}=\sqrt{\boldsymbol{F}^{T} \boldsymbol{F}}-\boldsymbol{I}
$$

where $\boldsymbol{U}$ is the right stretch tensor ${ }^{2}$. The stress-strain curves shown throughout this paper are obtained by tracking the evolution of $P_{z z}$ and $B_{z z}$, where $z$ is designated as the loading direction. For uniaxial loading simulations, $\dot{\boldsymbol{F}} \equiv \dot{\boldsymbol{F}}^{T}$ and thus $\dot{\boldsymbol{C}}=\dot{\boldsymbol{F}} \approx \dot{\boldsymbol{B}}$. We refer to the deformation rate represented by $\dot{F}_{z z}$ generically as $\dot{\varepsilon}$ in the remainder of this paper.

\footnotetext{
${ }^{2} \boldsymbol{U}$ emerges from the so-called polar decomposition: $\boldsymbol{F}=\boldsymbol{R} \boldsymbol{U}$, where $\boldsymbol{R}$ is a matrix the represents rigid rotation, and $\boldsymbol{U}$ is a pure stretch. Plasticity-induced crystal rotations are very important and give rise to crystallographic texture evolution in deformed crystals. However, only yielding is of concern here, and thus $\boldsymbol{U}$ is the component of interest.
} 


\subsection{The flow rule}

In the present $\mathrm{CP}$ calculations it is assumed that all the plastic deformation is due to dislocation slip. Then, the plastic velocity gradient can be written as:

$$
\boldsymbol{L}_{p}=\sum_{\alpha} \boldsymbol{P}_{\mathrm{S}}^{\alpha} \dot{\gamma}^{\alpha}
$$

where $\dot{\gamma}^{\alpha}$ is the slip rate on slip system $\alpha$, and $\boldsymbol{P}_{\mathrm{S}}^{\alpha}$ is a geometric projection tensor that will be defined later. The slip rate is calculated from Orowan's equation:

$$
\dot{\gamma}^{\alpha}=b \rho^{\alpha} v_{s}\left(\tau^{\alpha}, T\right)
$$

where $b=a_{0} \sqrt{3} / 2$ is the modulus of the Burgers vector, $a_{0}$ is the lattice parameter, $T$ the absolute temperature, $\rho^{\alpha}$ is the (mobile) screw dislocation density in slip system $\alpha$, and $v_{s}\left(\tau^{\alpha}, T\right)$ is the screw dislocation velocity. The present formulation of the flow rule belongs to the class of non-associated, rate-dependent $\mathrm{CP}$ models (McDowell, 2008).

The two characteristics that are particular to bcc plasticity are the thermally-activated nature of screw dislocation motion, which makes it the rate-controlling process during plastic deformation, and the existence of non-Schmid effects, i.e. deviations from the geometric projection law for the resolved shear stress. Both of these physical processes have been known for several decades, and have been carefully analyzed experimentally (cf. Section 1). If our intent is to predict the temperature dependence of the flow stress in bcc metals, accurate physical descriptions of both of them must be incorporated into our CP model. This is the subject of the following sections. As we shall see, non-Schmid effects establish the form of the projection tensor $\boldsymbol{P}_{\text {tot }}^{\alpha}$, while the velocity $v_{s}\left(\tau^{\alpha}, T\right)$ captures the thermally activated character of dislocation motion. We make tungsten the object of our study for a number reasons presented in previous works (Cereceda et al., 2013; Stukowski et al., 2015) ${ }^{3}$.

\subsubsection{Screw dislocation mobility law}

Except at high homologous temperatures and strain rates, screw dislocation motion is the rate-limiting step in bcc crystal deformation. Although recent dislocation dynamics simulations in $\alpha$-Fe challenge the notion that the dislocation density is monolithic across the entire temperature range (Monnet et al., 2009; Naamane et al., 2010; Monnet et al., 2011; Tang \& Marian, 2014), it is reasonable to assume a dominance of screw dislocations in the temperature and strain rate regimes considered in this work $\left(0<T / T_{m}<0.2\right.$ and $\dot{\varepsilon} \approx 10^{-4} \mathrm{~s}^{-1}$ ). In the thermally activated regime, screw dislocation motion proceeds via the nucleation of kink-pairs and their subsequent lateral relaxation. In the regime of interest here, kink relaxation is a significantly faster process than kink-pair nucleation, and it can thus be assumed that no new kink-pairs will be nucleated while lateral kink motion is underway (Stukowski et al., 2015). Such assumption leads to the following expression for the total time, $t_{t}$, required for a kink pair to form and sweep a rectilinear screw dislocation segment of length $\lambda^{\alpha}$ lying on a given slip plane:

$$
t_{t}=t_{n}+t_{k}=J\left(\tau^{\alpha}, T\right)^{-1}+\frac{\lambda^{\alpha}-w}{2 v_{k}\left(\tau^{\alpha}, T\right)}
$$

where $t_{n}$ is the mean time to nucleate a kink pair, $t_{k}$ is the time needed for a kink to sweep half a segment length, $J$ is the kink-pair nucleation rate, $w$ is the kink-pair separation, and $v_{k}$ is the kink velocity. The kink-pair nucleation rate follows an Arrhenius formulation (Stukowski et al., 2015):

$$
J\left(\tau^{\alpha}, T\right)=\frac{v_{0}\left(\lambda^{\alpha}-w\right)}{b} \exp \left(-\frac{\Delta H_{k p}\left(\tau^{\alpha}\right)}{k T}\right)
$$

\footnotetext{
${ }^{3} \mathrm{~W}$ is an elastic isotropic metal, which simplifies the constitutive plastic formulation.
} 
where $v_{0}$ is an attempt frequency, $\Delta H_{k p}$ is the activation enthalpy of a kink pair at stress $\tau^{\alpha}$, and $k$ is Boltzmann's constant. For its part, the kink velocity can be expressed as (Dorn \& Rajnak, 1964; Kocks et al., 1975):

$$
v_{k}\left(\tau^{\alpha}, T\right)=\frac{b \tau^{\alpha}}{B(T)}
$$

where $B$ is friction coefficient typically assumed to be linearly dependent on temperature. However, calculations made to obtain the value of $B$ for the interatomic potential employed in this work, have yielded no temperature dependence, and here $B$ is simply a constant (Swinburne et al., 2013) . The dislocation velocity can be obtained after operating with eqs. (13) and (14) as:

$$
v_{s}=\frac{h}{t_{t}}=\frac{h}{t_{n}+t_{k}}=\frac{2 b h \tau^{\alpha} v_{0}\left(\lambda^{\alpha}-w\right) \exp \left(-\frac{\Delta H_{k p}}{k T}\right)}{2 b^{2} \tau^{\alpha}+v_{0} B\left(\lambda^{\alpha}-w\right)^{2} \exp \left(-\frac{\Delta H_{k p}}{k T}\right)}
$$

where $h=a_{0} \sqrt{6} / 3$ is the distance between two consecutive Peierls valleys. We note that at low temperatures, or when $t_{k} \ll t_{n}$, the second term in the denominator vanishes and one recovers the standard diffusive velocity expression commonly used in crystal plasticity and dislocation dynamics:

$$
v_{s}=v_{0} h \frac{\left(\lambda^{\alpha}-w\right)}{b} \exp \left(-\frac{\Delta H_{k p}\left(\tau^{\alpha}\right)}{k T}\right)
$$

Figure 2: Schematic depiction of a kink pair on a screw segment of length $\lambda$ lying on a slip plane $\boldsymbol{n}^{\alpha}$ (of the $\{110\}$ family). The vertical axis represents the potential energy, with the Peierls potential clearly marked. The dashed line represents the initial equilibrium line position.

The parameterization of eq. (15) is a critical step that establishes a physical connection with the scales where kink-pairs are resolved as atomistic entities. This is the first essential piece of physics required to 
achieve predictive capabilities. We have devoted much effort in past works to calculate the necessary parameters from fundamental models based on semiempirical interatomic potentials (Cereceda et al., 2013; Stukowski et al., 2015). The list of parameters employed in this work and their associated values and units are given in Table 1. The physical meaning of some of these parameters is best expressed in pictorial form. Figure 2 shows a schematic diagram of the topology of a kink pair lying on the Peierls energy substrate. The figure highlights the physical meaning of each parameter listed in the table. In addition to the references provided earlier, a detailed description of the protocols used to calculate all the adjustable parameters in our formulation is provided by Cereceda (2015).

At this stage, it is worth to introduce a note about the available slip systems (which establish the running indices of $\alpha$. Stukowski et al. (2015) have shown that in W an elementary glide on a $\{112\}$ plane is a composite of two elementary steps on alternate $\{110\}$ planes. Judging by these results, we conclude that glide on any given plane is achieved by way of sequential $\{110\}$ jumps, which constitutes the basis to simulate plastic yielding in the foregoing Sections. This is consistent with recent atomistic simulations (Cereceda et al., 2013) and experiments (Caillard, 2010a,b; Marichal et al., 2013, 2014) and limits the number of available slip systems in our study to 12 (listed in Appendix A). We note that this model of slip for $\mathrm{W}$ is not necessarily suggestive of what may happen in other bcc crystals (Franciosi et al., 2015).

\subsubsection{Projection tensor and non-Schmid effects}

The tensor $\boldsymbol{P}_{\mathrm{S}}^{\alpha}$ introduced in eq. (10) represents the Schmid (geometric) projection of the strain rate contribution from a slip system defined by the plane normal $\boldsymbol{n}^{\alpha}$ and slip direction $\boldsymbol{m}^{\alpha}$ (both unit vectors). However, as pointed out above, $\boldsymbol{P}_{\mathrm{S}}^{\alpha}$ does not capture the full panoply of non-Schmid effects needed to calculate the value of the resolved shear stress on that slip system, $\tau^{\alpha}$. For this, we introduce a total projection tensor $\boldsymbol{P}_{\text {tot }}^{\alpha}$ such that:

$$
\tau^{\alpha}=\boldsymbol{P}_{\mathrm{tot}}^{\alpha}: \sigma=\left(\boldsymbol{P}_{\mathrm{S}}^{\alpha}+\boldsymbol{P}_{\mathrm{T} / \mathrm{AT}}^{\alpha}+\boldsymbol{P}_{\mathrm{ng}}^{\alpha}\right): \sigma
$$

where

$$
P_{\mathrm{S}}^{\alpha}=\boldsymbol{m}^{\alpha} \otimes \boldsymbol{n}^{\alpha}
$$

is the Schmid tensor, with

$$
\sigma=J^{-1} \boldsymbol{F} \boldsymbol{S} \boldsymbol{F}^{T}
$$

the Cauchy (true) stress and $J=\operatorname{det}(\boldsymbol{F})$ the Jacobian. The tensors

$$
\begin{aligned}
\boldsymbol{P}_{\mathrm{T} / \mathrm{AT}}^{\alpha} & =a_{1} \boldsymbol{m}^{\alpha} \otimes \boldsymbol{n}_{1}^{\alpha} \\
\boldsymbol{P}_{\mathrm{ng}}^{\alpha} & =a_{2}\left(\boldsymbol{n}^{\alpha} \times \boldsymbol{m}^{\alpha}\right) \otimes \boldsymbol{n}^{\alpha}+a_{3}\left(\boldsymbol{n}_{1}^{\alpha} \times \boldsymbol{m}^{\alpha}\right) \otimes \boldsymbol{n}_{1}^{\alpha}
\end{aligned}
$$

are non-Schmid tensors representing respectively the twinning/anti-twinning asymmetry (T/AT) and the effects due to non-glide stress components. $a_{1}, a_{2}$, and $a_{3}$ are material-dependent constants that must also be calculated and added to our parameterization database. The vector $\boldsymbol{n}_{1}^{\alpha}$ forms an angle of $-60^{\circ}$ with the reference slip plane defined by $\boldsymbol{n}^{\alpha}$, and changes sign with the direction of slip on each glide plane (Koester et al., 2012).

The present non-Schmid formulation was originally developed by Vitek and expanded by others, and has been successfully used to propose yielding criteria adapted to finite element and crystal plasticity calculations in a number of cases (Gröger \& Vitek, 2008; Chen et al., 2013; Weinberger et al., 2012). The reader is referred to these works for more details but it is worth pointing out that the methodology that these authors have proposed is not unique, and that other rigorous implementations of non-Schmid effects could equally be devised. For the purposes of this section, suffice it to say that the particularities of the screw dislocation core and the bcc lattice structure result in deviations from a purely geometric projection. These deviations originate, respectively, from a geometric asymmetry between the twinning and anti-twinning directions of the $\langle 111\rangle$ zone -from which $a_{1}$ is first calculated-, and from the effect that nonglide components (termed generically ' $\sigma$ ') of the local stress tensor have on the critical resolved shear stress, from which $a_{2}$ and $a_{3}$ are obtained. Atomistic calculations specifically designed to calculate the non-Schmid critical stress $\tau_{c}^{\chi}$ 
as a function of the angle $\chi$ between the maximum resolved shear stress (MRSS) plane were performed according to the geometry shown schematically in Figure 3. The Figure shows the mapping between the atomistic box and the crystallography of the [111] zone. Following the sign convention used in the Figure, the stress tensor applied is:

$$
\left(\begin{array}{ccc}
-\sigma & 0 & 0 \\
0 & \sigma & \tau \\
0 & \tau & 0
\end{array}\right)
$$

which activates axial (nonglide) stress components while maintainign zero pressure. $\tau_{c}^{\chi}$ is expressed as a

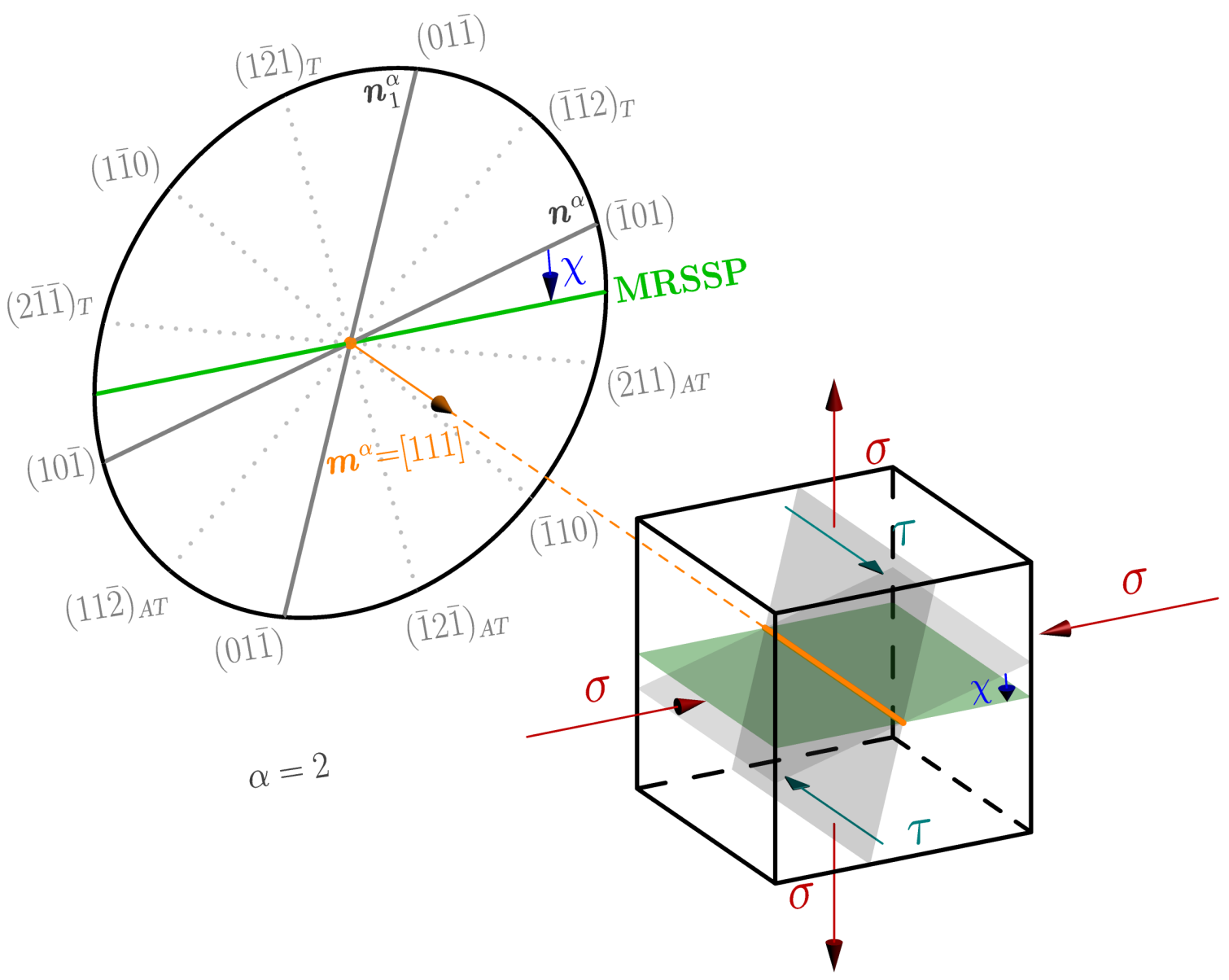

Figure 3: Crystallographic diagram of the [111] zone in the bcc lattice with each $\{110\}$ and $\{112\}$ clearly labeled. The picture also shows a mapping of the [111] zone to a schematic atomistic box containing a screw dislocation subjected to shear and nonglide stresses according to Vitek's convention. This setup is used to calculate the critical RSS using atomistic calculations (cf. Appendix B). The glide $\boldsymbol{n}^{\alpha}$, auxiliary $\boldsymbol{n}_{\mathbf{1}}{ }^{\alpha}$ and MRSS planes are labeled in each case. A [101] glide plane corresponds to $\alpha=2$ in our CP calculations.

combination of the contributions displayed in Fig. 3:

$$
\tau_{c}^{\chi}=\frac{\tau_{c}^{*}+\sigma\left(a_{2} \sin (2 \chi)+a_{3} \sin \left(2 \chi+\frac{\pi}{6}\right)\right)}{\cos \chi+a_{1} \cos \left(\chi+\frac{\pi}{3}\right)}
$$

where $\tau_{c}^{*}$ is a fitting constant that represents the Peierls stress. The details of these atomistic calculations are provided in Appendix B. The results for $\tau_{c}^{\chi}$ are shown in Figure 4 as a function of $\chi$ and $\sigma$, with $\tau_{c}^{*}, a_{1}$, 
$a_{2}$, and $a_{3}$ given in Table 1. It is worth noting that the relation between $\tau_{c}^{\chi}$ and $\sigma$ has been established for tensile nonglide stresses only $(\sigma>0)$, for consistency with the linear dependence used in the work of Vitek and collaborators (Gröger et al., 2008a,b) that has been used in other crystal plasticity works (Koester et al., 2012). However, nothing precludes the use of nonlinear fitting functions that capture both the tensile and compressive regimes simultaneously (cf. Appendix B). It is worth noting that Gröger et al. (2008b) obtained values of $a_{1}=0, a_{2}=0.56$, and $a_{3}=0.75$ using a bond-order potential, substantially far from our values for those parameters.

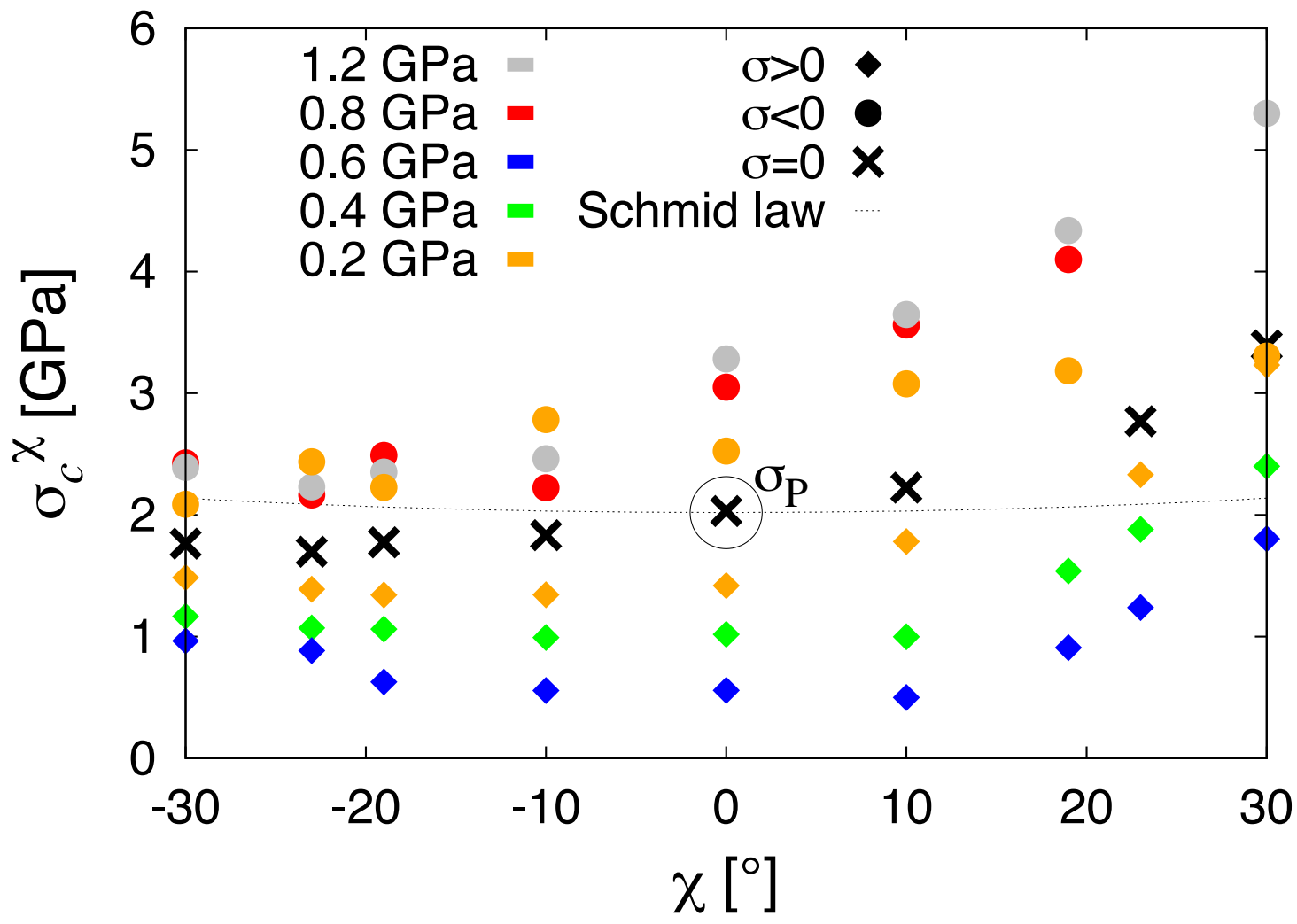

Figure 4: Critical resolved shear stress as a function of the angle $\chi$ between the MRSS and glide planes and the value of the nonglide stress component $\sigma$ with the sign convention according to Fig. 3. The value of the Peierls stress $\sigma_{P}=2.03$ GPa is circled.

By way of example, we calculate the maximum projection factor $M$ for directions in the standard stereographic triangle using the fully parameterized projection tensor:

$$
M=(\boldsymbol{l} \otimes \boldsymbol{l}): \boldsymbol{P}_{\mathrm{tot}}=(\boldsymbol{l} \otimes \boldsymbol{l}):\left(\boldsymbol{P}_{\mathrm{S}}^{\alpha}+\boldsymbol{P}_{\mathrm{T} / \mathrm{AT}}^{\alpha}+\boldsymbol{P}_{\mathrm{ng}}^{\alpha}\right)
$$

where $l$ is the loading direction, which is obtained by visiting each of the nodes resulting from the discretization of the standard triangle area into a uniform grid consisting of 231 points. The results for tension $(\sigma>0)$ are shown in Figure 5. It is clear than non-Schmid effects -particularly the impact of nonglide componentsare critical to calculate the RSS on a given slip system. We find that from a maximum nominal value of $M=0.5$ for the standard Schmid law $\left(\boldsymbol{P}_{\mathrm{S}}^{\max }\right)$ there is a twofold amplification when the twinning/antitwinning asymmetry is considered $\left(\boldsymbol{P}_{\mathrm{S}}^{\max }+\boldsymbol{P}_{\mathrm{T} / \mathrm{AT}}^{\max }\right)$, and an astonishing fourfold increase when nonglide effects are also included $\left(\boldsymbol{P}_{\mathrm{S}}^{\max }+\boldsymbol{P}_{\mathrm{T} / \mathrm{AT}}^{\max }+\boldsymbol{P}_{\mathrm{ng}}^{\max }\right)$. As we shall see in Section 3.2, this has extraordinary importance when comparing $\mathrm{CP}$ calculations to experimental measurements. 


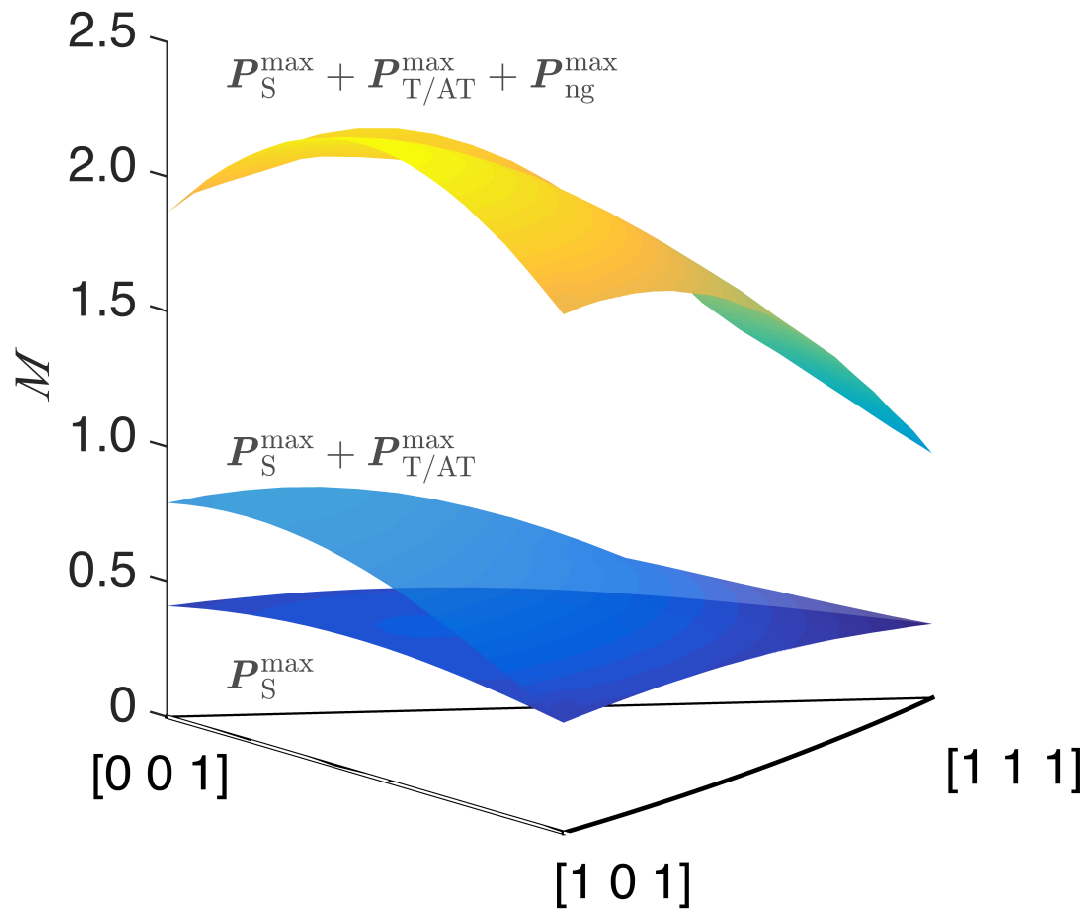

Figure 5: Projection factor according to eq. (22) for 231 directions within the standard triangle. The contributions of each of therms in eq. (16) are broken down for comparison.

\subsection{Dislocation density evolution model}

To close the model, one needs to provide an evolution law for the dislocation density in Orowan's equation 11. There are numerous density evolution models proposed in the literature, each with a specific domain of applicability (Mecking \& Kocks, 1981; Estrin, 1996; Arsenlis \& Parks, 2002; Stainier et al., 2002; Barton et al., 2011). In this work we are mainly interested in yielding, i.e. the elastic-to-plastic transition before dislocation-based slip takes on a dominant role in the constitutive model. We use the model presented by Roters (2011), in which the mobile dislocation density on slip system $\alpha$ evolves in time according to:

$$
\dot{\rho}^{\alpha}=\dot{\rho}_{\text {mult }}^{\alpha}+\dot{\rho}_{\text {ann }}^{\alpha}
$$

The evolution model is initialized by the dislocation density at $t=0, \rho_{0}^{\alpha}$. In eq. (23), $\dot{\rho}_{\text {mult }}^{\alpha}$ and $\dot{\rho}_{\text {ann }}^{\alpha}$ represent the dislocation multiplication and dislocation annihilation rate terms, respectively. In this model, both $\dot{\rho}_{\text {mult }}^{\alpha}$ and $\dot{\rho}_{\text {ann }}^{\alpha}$ are directly proportional to the plastic strain rate. Dislocation multiplication is treated as being proportional to the inverse mean free path of the dislocations, $\lambda_{\alpha}$ :

$$
\dot{\rho}_{\text {mult }}^{\alpha}=\frac{\left|\dot{\gamma}^{\alpha}\right|}{b \lambda^{\alpha}}
$$


which is defined as a function of the grain size $d_{g}$, the forest dislocation density $\rho_{f}^{\alpha}$, and a hardening constant $c$ :

$$
\frac{1}{\lambda^{\alpha}}=\frac{1}{d_{g}}+\frac{\sqrt{\rho_{f}^{\alpha}}}{c}
$$

Here, $c$ and $d_{g}$ are set, respectively, to one and to an arbitrarily high value such that the term controlling the dislocation mean free path is:

$$
\lambda^{\alpha} \approx\left(\sqrt{\rho_{f}^{\alpha}}\right)^{-1}
$$

${ }^{4}$ Although the $\xi_{\alpha \alpha^{\prime}}$ coefficients were calculated for bcc Fe and not W, the results are equally applicable because Fe was treated as isotropic elastic -as is $\mathrm{W}$ - and the interaction matrix coefficients are non-dimensional and independent of the value of the plastic constants considered. 
Table 1: List of parameters and functional dependences for fitting the CP model. All of these parameters have been obtained using dedicated atomistic calculations. The parameter $s$ represents the normalized shear stress: $s=\frac{\tau^{\prime \alpha}}{\sigma_{P}}$ (cf. eq. (28)).

\begin{tabular}{llc}
\hline parameter & value or function & units \\
\hline$a_{0}$ & 3.143 & $\AA$ \\
$b$ & 2.72 & $\AA$ \\
$h$ & $a_{0} \sqrt{6} / 3$ & $\AA$ \\
$\mathbb{C}_{11}$ & 523 & $\mathrm{GPa}$ \\
$\mathbb{C}_{12}$ & 202 & $\mathrm{GPa}$ \\
$\mathbb{C}_{44}$ & 161 & $\mathrm{GPa}$ \\
$v_{0}$ & $9.1 \times 10^{11}$ & $\mathrm{~s}$ \\
$\sigma_{P}$ & 2.03 & $\mathrm{GPa}$ \\
$B$ & $8.3 \times 10^{-5}$ & $\mathrm{~Pa} \cdot \mathrm{S}$ \\
$\Delta H(s ; T)$ & $\Delta H_{0}\left(1-s^{p}\right)^{q}$ & $\mathrm{eV}$ \\
$\Delta H_{0}$ & 1.63 & $\mathrm{eV}$ \\
$p$ & 0.86 & - \\
$q$ & 1.69 & - \\
$w$ & 11 & $b$ \\
$\sigma_{c}^{\chi}$ & $\frac{\tau_{c}^{*}+\sigma\left(a_{2} \sin (2 \chi)+a_{3} \sin (2 \chi+\pi / 6)\right)}{\cos \chi+a_{1} \cos (\pi / 3+\chi)}$ & $\mathrm{GPa}$ \\
$a_{1}$ & 0.938 & - \\
$a_{2}$ & 0.71 & - \\
$a_{3}$ & 4.43 & - \\
$\tau_{c}^{*}$ & 2.92 & $\mathrm{GPa}$ \\
$c$ & 1 & - \\
$d_{g}$ & 2.72 & $\AA$ \\
$d_{\text {edge }}$ & 2.72 & $\AA$ \\
\hline
\end{tabular}

Table 2: Values of $\xi_{\alpha \alpha^{\prime}}$ for latent hardening in bcc crystals (from Queyreau et al. (2009)).

\begin{tabular}{|cccccc|}
\hline self & coplanar & collinear & orthogonal & glissile & sessile \\
\hline 0.009 & 0.009 & 0.72 & 0.05 & 0.09 & 0.06 \\
\hline
\end{tabular}

\subsection{Yield criterion}

In metals, where dislocation flow is not a singular event but a diffuse continuous process, it is generally accepted that the definition of yield point ${ }^{5}$ is not unique. Perhaps as the result of these conceptual indetermination, modern usage has evolved into that of an arbitrary rule, the $0.2 \%$ strain offset rule for obtaining the yield stress of metals. For materials having nonlinear elastic behavior, there are not even arbitrary rules, only individual preferences and proclivities in defining yield when a given amount of strain has been reached. It is quite apparent then that to define robust yield criteria it is necessary that they be implemented and supported by consistent and meaningful definitions in terms of the stress-strain behavior. This is often difficult when the transition from the elastic to the inelastic regimes is obscured in the global picture of deformation. However, in the present calculations we effectively possess an arbitrary degree of data resolution and can define an unambiguous mathematical criterion.

The preferred method for defining the elastic limit of a ductile material is to compute the second derivative of the stress-strain curve, referred to generically as $\sigma(\varepsilon)$, and identify the location of the inflection

\footnotetext{
${ }^{5}$ Also referred to as elastic limit, proportionality limit, yield stress, etc.
} 
point (Christensen, 2008). The yield point then corresponds to the strain, $\varepsilon_{y}$, for which $\left|\frac{d^{2} \sigma}{d \varepsilon^{2}}\right|$ is maximum. Mathematically:

$$
\sigma_{y}=\sigma\left(\varepsilon_{y}\right), \varepsilon_{y}:=\varepsilon|\max | \frac{d^{2} \sigma}{d \varepsilon^{2}} \mid
$$

For ductile metals, the location of the maximum of the second derivative represents the point at which dislocation-mediated flow is the major contribution to $L$ (cf. Section 2.1). However, this condition works surprisingly well for other materials such as glassy polymers, where flow might be caused by molecular rearrangement and damage at both the molecular and macroscopic scales (Bowden \& Jukes, 1972).

To illustrate the accuracy of the second-derivative method, we plot in Figure 6 the first and second derivative of a stress-strain curve corresponding to a [101] uniaxial tensile test of a W single crystal under representative initial conditions. Recall from Section 2.2 that the stress and strain metrics of choice are $P$ and $\boldsymbol{B}$, and so we plot $\frac{d P_{z z}}{d B_{z z}}$ and $\frac{d^{2} P_{z z}}{d B_{z z}^{2}}$ specifically. The inflection point -marked by a vertical dashed line in

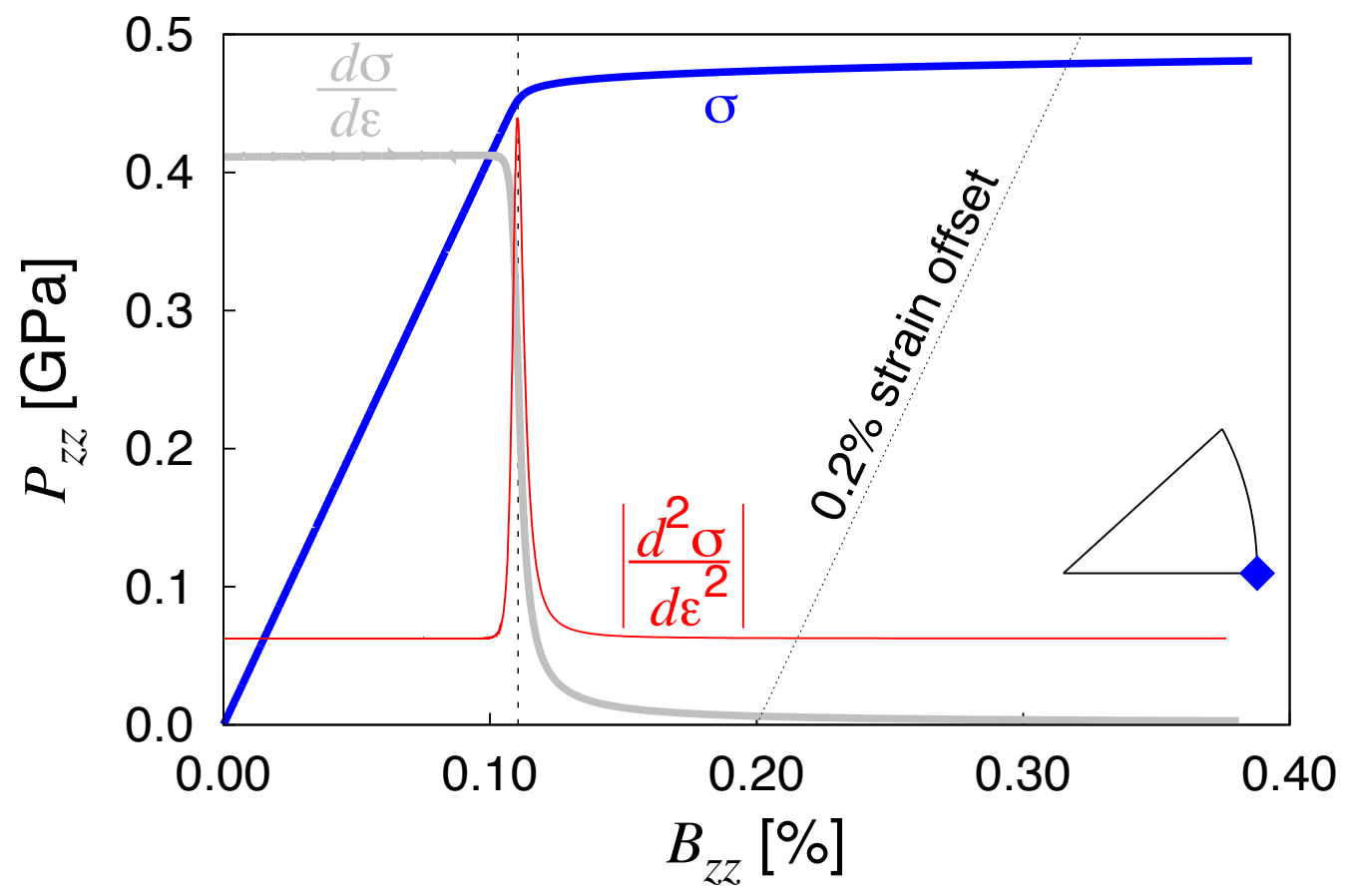

Figure 6: Evolution of the stress $P_{z z}$ with deformation $B_{z z}$ during a CP simulation of a uniaxial tensile test with [101] loading orientation (as depicted in the standard triangle). The first and second derivatives of the stress w.r.t. to the strain are also plotted to illustrate the method of identification of the yield point according to this criterion. Also shown is the intercept of the curve with the $0.2 \%$ strain offset criterion line.

the figure- occurs for $\varepsilon_{y}=0.1105 \%$, for which a value of $\sigma_{y}=0.452 \mathrm{GPa}$ is obtained. The figure also shows the $0.2 \%$ strain offset criterion, which -by contrast- gives $\varepsilon_{y}=0.3167 \%$ and $\sigma_{y}=0.479 \mathrm{GPa}$, i.e. a three-fold difference in strain and approximately a $6 \%$ difference in stress with respect to the stress second derivative criterion.

However, determining the first and second derivatives of the stress-strain relation can become numerically intensive, especially when evaluating thousands of curves as is the case in this work. An approximation to this method that works particularly well for linear-elastic materials that display a clear elastic-to-plastic 
transition is to take the yield point as the first point in the $\sigma(\varepsilon)$ function that satisfies:

$$
\frac{d \sigma}{d \varepsilon}<E(1-\delta)
$$

i.e. $\sigma_{y}$ is measured as the stress for which a departure from linearity (as set by the elastic regime) larger than some small value $\delta$ is observed in the stress-strain relation. We have found that a value of $\delta \approx 0.01$ is sufficient to predict the value of $\sigma_{y}$ within a small error relative to the value furnished by the secondderivative method. By way of example, for the curve shown in Fig. 6 and $\delta=0.01$, we find a values of $\varepsilon_{y}=0.1055 \%$ and $\sigma_{y}=0.435 \mathrm{GPa}$, or less than a $4 \%$ difference with the numbers according to the secondderivative criterion. With this reasonable accuracy and the computational advantages alluded to above, we then use the $\delta=0.01$ criterion in the remainder of this paper.

\subsection{Model validation and initial results}

Prior to deploying our fully-parameterized CP method for numerically-intensive calculations, it is essential to undergo a thorough exercise of validation. Experimental data from uniaxial tensile tests in single crystal W at low strain rates are scant and sporadic, with the main sources listed below:

1. Argon \& Maloof (1966) performed some early experiments at a strain rate of $10^{-4} \mathrm{~s}^{-1}$ and temperatures of 77, 199, 293, 373, and $450 \mathrm{~K}$. These authors measured the yield strength for the three vertices of the stereographic triangle [001], [110], and [111] with an initial dislocation density of $\rho_{0} \approx 10^{10}$ $\mathrm{m}^{-2}$.

2. Raffo (1969) analyzed the yielding behavior of arc-melted W between 77 and $680 \mathrm{~K}$ at $\dot{\varepsilon}=8.3 \times 10^{-4}$ $\mathrm{s}^{-1}$. However, the loading orientation is not given and most of the tests were done in compression.

3. Stephens (1970) has carried out compression tests at 150, 300, and $590 \mathrm{~K}$. This researcher focuses on dislocation density evolution and dislocation substructures, however, with a value of $\rho_{0} \approx 1.4 \times 10^{14}$ $\mathrm{m}^{-2}$, notably larger than in other tests. There have been other works that have also focused mainly on compression tests (Klopp et al., 1964; Gupta \& Li, 1970).

4. Brunner $(2000,2010)$ has performed a series of experiments more recently at temperatures between 77 and $800 \mathrm{~K}$. They employed a value of $\dot{\varepsilon}=8.5 \times 10^{-4} \mathrm{~s}^{-1}$ and loaded the system uniaxially along the [1 149$]$ direction with a starting dislocation density of $5.5 \times 10^{9} \mathrm{~m}^{-2}$.

As pointed out in Section 2.3.2, our CP model is parameterized for tensile tests only and so for validation we focus on the works by Argon \& Maloof (1966) and Brunner (2000, 2010). Argon \& Maloof (1966) centered on multislip by considering mainly loading orientations coincident with the vertices of the standard triangle. Consequently, we replicate their test conditions in our CP model and compare the results obtained by taking into account all the different elements of the projection tensor (16). The results are shown in Figure 7 for the [111] and the [110] loading orientations, with the insets in both figures showing the relative importance of considering each of the non-Schmid contribution to the projection tensor incrementally. While our calculations are in general good agreement with the [111] test data, they deviate from the experimental results at the two lower temperature points for the [110] orientation. Argon \& Maloof (1966) point out that, at low temperatures, deformation by twinning may play a larger role when loading along [110] relative to other orientations. This may be at the origin of the discrepancy, as twinning is not part of the catalog of deformation mechanisms considered in this model.

Next we simulate uniaxial tensile tests under single slip conditions, i.e. along crystal orientations near the center of the standard triangle. This corresponds to the experiments by Brunner $(2000,2010)$ referred to above, which were done more recently with more advanced instrumentation. The results are shown in Figure 8 , where we also show the curves using the different elements of eq. (16). This time, the agreement is striking, particularly again at temperatures above $400 \mathrm{~K}$. Specifically, the athermal limit $(\approx 710 \mathrm{~K})$ is particularly well reproduced, as is the extrapolated critical stress at $0 \mathrm{~K}$ (Peierls stress), which is within $10 \%$ of the experimental values.

Although, as noted earlier, the main focus of this work is on yielding, we have applied the fully parameterized model to study the flow stress regime for some selected cases in Appendix C. The results shown 
there demonstrate the performance of the method outside the primary range of application. While the model cannot be assumed to be predictive in the post-yield regime under general loading conditions, these are encouraging results that strengthen the notion that parameter-free $\mathrm{CP}$ calculations can perform well under specific deformation scenarios.

With the confidence conferred on our CP model by the validation procedure, next we proceed to calculate the yield strength for a number of numerically-intensive scenarios. This is the object of the following sections.

\subsection{Uniaxial tensile tests}

In this Section, we report on the uniaxial yielding results as a function of temperature and strain rate. Our results are organized by strain rate, such that we first provide a detailed account of all the calculations at a given strain rate followed by a study on the dependence with $\dot{\varepsilon}$.

\subsubsection{Results at $\dot{\varepsilon}=10^{-3} \mathrm{~s}^{-1}$}

For these calculations, we have discretized the area of the standard triangle into a uniform grid consisting of 231 nodes, each representing a crystallographic loading orientation. We begin with calculations at a prescribed strain rate of $\dot{\varepsilon}=10^{-3} \mathrm{~s}^{-1}$. Figure 9 shows colored contour plots of the yield stress in the 100-to-600 K temperature range. Areas of high relative yield strength can be seen to concentrate around the vertices of the standard triangle, representing multislip conditions, whereas soft regions develop in two distinct locations of the triangle, one near the [324] zonal axis that then rotates towards [112] above 500 $\mathrm{K}$, and another near [102]. Note that, to accentuate the differences between hard and soft regions, each contour plot has its own specific numerical scale.

We have extracted the specific location of the global extrema in the standard triangle and plot it as a function of temperature in Figure 10(a). The hardest direction is consistently the [101], while the softest is seen to revolve around the vicinity of the [112] axis, first along [30 18 41] at $100 \mathrm{~K}$, then along [180 131 271] between 200 and $500 \mathrm{~K}$, and finally rotating towards [9 934 ] for $T>500 \mathrm{~K}$. Next, we plot the detailed temperature dependence of the yield strength corresponding to the hardest and softest directions -as given by Fig. 10(a)- for this strain rate in Figure 11. As the calculated data show, there is approximately a 30\% difference in yield stress between the hardest and softest directions. Interestingly, this gap appears to be fairly independent of temperature. Above $650 \mathrm{~K}$, the curves begin to level off, signaling the onset of the athermal regime.

\subsubsection{Dependence on strain rate and strain rate sensitivity}

In this Section we expand the analysis presented in the previous Section to strain rates of $10^{-4}$ and $10^{-5} \mathrm{~s}^{-1}$. To avoid redundancies, here we show only the temperature trajectory of the softest and hardest loading orientations in Figs. 10(b) and 10(c), which emanate from calculations as those presented in Fig. 9. The results are quantitative similar to the case of $\dot{\varepsilon}=10^{-3} \mathrm{~s}^{-1}$, with the only appreciable deviations occurring at temperatures above $450 \mathrm{~K}$. At these high temperatures, the softest orientation rotates clearly towards the vicinity of the [113] zonal axis, without excursions near [103] as was the case for the $\dot{\varepsilon}=10^{-3}$ calculations.

As above, we add the temperature dependence of the yield stress for the hardest and softest directions at these strain rates to Figure 11. The data show the same qualitative trend for all strain rates, with the same approximate $30 \%$ difference between the hard and soft orientations. However, useful information can be extracted if the strain-rate dependence of the yield stress is plotted for selected orientations. Then, one can calculate the so-called strain rate sensitivity, characterized by the strain rate sensitivity exponent $m$, of the material as a function of temperature. Strain rate sensitivity is exceedingly important to delay the onset of inhomogeneous deformation (Hutchinson \& Neale, 1977), e.g. necking, and is used as a criterion to assess the possibility of superplastic behavior in certain kinds of materials (Hedworth \& Stowell, 1971; Arieli \& Rosen, 1976). This belongs more in the realm of failure and is thus outside the scope of this paper. However, it is of interest to calculate the strain rate sensitivity of the yield stress and relate our findings to the larger failure picture if possible. 
This precisely what is done in Figure 12 for [101] loading tests. The figure shows the variation of the yield strength at the three strain rates considered here, again in the range $100<T<600 \mathrm{~K}$. The data can 3 then be fitted to the following expression:

$$
\sigma_{y}=C \dot{\varepsilon}^{m}
$$

where $C$ is a fitting constant. The strain rate sensitivity exponent is formally defined as:

$$
m=\frac{\partial \log \sigma_{y}}{\partial \log \dot{\varepsilon}}
$$

$m$ is plotted in the inset to Fig. 12, where it can be seen that it increases monotonically with temperature from a value of $m=0.01$ at $100 \mathrm{~K}$ to $\approx 0.2$ at $600 \mathrm{~K}$. The implications of these results will be discussed in Section 4

\subsection{Biaxial loading tests and yield surfaces}

For non-associated CP formulations such as the present one, yielding is not a separate and independent criterion, but a consequence of the constitutive law of the material behavior (Bodner, 1968). Indeed, with yielding defined on the basis of the identification criterion introduced in Section 3.1, yield surfaces are furnished as a product of the $\mathrm{CP}$ calculations. In this Section we calculate the yield curves under biaxial stress conditions for selected pairs of orthogonal loading directions $l_{y}$ and $l_{z}$. As noted in Section 2.3.2, the present implementation of the non-Schmid stress projection law is only valid for tensile conditions ${ }^{6}$. Thus, our yield curves are only meaningful in the positive stress quadrant (or octant, for yield surfaces). The procedure to calculate each point of the yield surface consists of deforming the system simultaneously along the prescribed orientations until the material yields on either one according to criterion (29). The stresses $P_{z z}$ and $P_{y y}$ are then measured along both directions and the resulting duplet is added to the curve. Plane stress conditions are adopted along the remaining direction, i.e. $P_{x x}=0$. The calculations are done at a nominal strain rate of $\dot{\varepsilon}=10^{-4} \mathrm{~s}^{-1}$, with slight variations above and below this value in one of the loading directions to accumulate different levels of stress and map the entire stress quadrant.

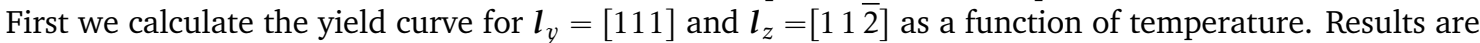
shown in Figure 13. The curves enclose domains that are everywhere convex, thus satisfying the DruckerPrager criterion for stable plastic flow materials (Prager, 1952; Drucker et al., 1952). The absolute values and the temperature sensitivity of the yield stresses for the end cases of $P_{z z}=0$ and $P_{y y}=0$ are consistent with the results shown in Section 3.3 for the $\boldsymbol{l}_{y}$ and $\boldsymbol{l}_{z}$ chosen here.

The next series of calculations involves determining the entire yield surface of the [111] zone, i.e. for a set of directions orthogonal to [111] in $10^{\circ}$ intervals, at a fixed temperature of $300 \mathrm{~K}$. Results are shown in Figure 14. Symmetry considerations limit the angular range to be explored to a $60^{\circ}$ arc, which is shown in the figure. Yield surfaces such as this one are the culmination of crystal plasticity calculations, and can be used as constitutive input into continuum models to simulate effective mechanical behavior at the engineering scale, for component design and/or to simulate, e.g., thermo-mechanical treatments (Sheng et al., 2004; Serenelli et al., 2010).

\section{Discussion and conclusions}

In this Section we consider the most important implications of our results. First, we discuss one of the most salient characteristic of the current work. The present CP model uses a standard rate-dependent, finite-deformation, non-associated theory of crystal plasticity. However, while the underlying kinematic formulation serves as the mathematical framework upon which to build a physical methodology, it is via the connection to the material physics that the model is rendered truly predictive. Our technique does so by incorporating the following three features of bcc slip:

\footnotetext{
${ }^{6}$ Although this is not a limitation in a strict sense as it is done simply for consistency with non-Schmid treatments published in the literature.
} 
- A complete (T/AT plus nonglide) treatment of non-Schmid effects.

- A kinematic flow rule based on a thermally-activated screw dislocation mobility.

- Using accurate interatomic potentials for computing all the free parameters in the model.

We have shown that the full model is capable of predicting the experimentally-measured temperature dependence of yield strength in the entire temperature range for $\mathrm{W}$ single crystals without parameter-fitting of any kind ${ }^{7}$. The sole source of material (constitutive) information is a carefully selected semi-empirical interatomic potential fitted exclusively to a DFT-generated dataset that includes the Peierls stress in its full atomistic meaning. This closes the gap seemingly separating electronic structure calculations of fundamental dislocation core properties and real measurements of the yield stress in uniaxial tensile tests of bcc materials.

Indeed, much effort has been devoted to the study of this long-standing experiment/simulation discrepancy, particularly at temperatures $<20 \mathrm{~K}$. Explanations based on collective dislocation dynamics, such as network kinetics (Bulatov \& Cai, 2002) and/or mutually interacting dislocations (Gröger \& Vitek, 2007) can be more or less discounted in light of recent detailed electron microscopy experiments of isolated screw dislocation motion (Caillard, 2010a,b, 2014). A more recent description, based on quantum effects at very low temperatures, has been put forward with reasonable success (Proville et al., 2012). On this basis, our first partial conclusion is that, while the present calculations do not provide sufficient grounds to invalidate these theories, they do clearly demonstrate that models based solely on classical mechanics -and without recourse to fitting to experimental results- can be formulated to predict the temperature dependence of the yield strength of bcc single crystals. Evidently, we issue this conclusion with caution, as W does not constitute by itself a representative sample to convincingly claim generality, but we believe that it constitutes a step in that direction.

Another important physical aspect of tensile deformation in single bcc crystals is the seemingly distinct slip mechanisms operating in different temperature ranges. According to Seeger and collaborators, there are three clearly distinguishable temperature regions in the flow stress-temperature curves for bcc metals (Seeger, 1981, 1995; Brunner, 2000), namely, the so-called upper and lower bend temperatures, $\check{T}$ and $\hat{T}$, and the knee temperature $T_{k}{ }^{8} . \check{T}, \hat{T}$, and $T_{k}$ delimit three different regimes where slip may occur on $\{110\}$, as well as $\{112\}$, glide planes, and give rise to different deformation mechanisms. Although these theories are substantiated by ample experimental data, there are recent studies that indicate that $\{110\}$ slip may be sufficient to explain the most salient features of bcc plasticity (Marichal et al., 2013; Ali et al., 2011). This is consistent with the analysis presented here, backed by atomistic input, which suggests that only $\{110\}$ slip is admissible in bcc W. Interestingly, the screw dislocation mobility law employed in this work, where $\{112\}$ slip is disallowed by construction (cf. Section 2.3.1), is sufficient to quantitatively characterize the evolution of the yield stress across the entire temperature spectrum, without any ad hoc partition of mechanisms into different temperature regimes. We emphasize once more that the screw mobility law has been fitted exclusively to first-principles data.

In Section 3.3.2 we have provided calculations of the strain rate sensitivity defined as $m=\partial \log \sigma_{y} / \partial \log \dot{\varepsilon}$. It must be noted that our value of $m=0.023$ at $300 \mathrm{~K}$ obtained in the $10^{-3}>\dot{\varepsilon}>10^{-5} \mathrm{~s}^{-1}$ range is consistent with measurements performed by Zurek \& Gray III (1991) in W compressed uniaxially at strain rates from $10^{-3}$ to $10^{3} \mathrm{~s}^{-1}$. Notwithstanding the differences in experimental methodology and strain rate regime, this is also encouraging agreement for a result other than yield. $m$ is an important parameter for calculating the kink-pair activation enthalpy and activation volume from stress-relaxation tests. Note that some authors use an alternative definition for the strain rate sensitivity (Raffo, 1969; Brunner, 2000), namely, $\lambda=\partial \sigma / \partial \log \dot{\varepsilon}$, which is related to $m$ via $\lambda=m \sigma$. We can then conclude that the agreement achieved

\footnotetext{
${ }^{7}$ Of course, interatomic potentials -which form the basis of the constitutive information employed here- are subjected to a fair amount of fitting themselves, both to experimental data and first-principles calculations. However, potential fitting is extraneous to our work, in the sense that it was neither performed by us nor done with this application in mind, while the parameter fitting that we refer to here is dedicated specifically to reproduce experimental data of interest to the application of the model.

${ }^{8} T_{k}$ is understood as the temperature above which the contribution of the kink-pair formation mechanism to the flow stress becomes negligibly small, i.e. it signals the athermal limit.
} 
for a derivative quantity of the yield stress such as $m$ is symptomatic of the quality of the method outside the primary validation space.

The advantages of this and other $\mathrm{CP}$ methodologies w.r.t. more accurate techniques such as molecular dynamics, dislocation dynamics, or phase field methods is of course their computational expediency. Backed by the encouraging outcome of the validation exercise, this has enabled us to map the entire loading orientation space in the standard triangle (231 directions) as a function of temperature in a experimentally-meaningful strain rate range. These results can then be used to extract useful information, such as the strongest and softest orientations as a function of temperature and strain rate, or the strain rate sensitivity of our W model system. This information can ultimately be used to define yield criteria under a variety of conditions for more homogenized methods, with the aim put on component design.

In this sense, the culmination of the CP simulations is the calculation of yield curves and yield surfaces in stress space. The stress space that we have chosen for our yield surface calculations is a purely biaxial one (in plane stress) with one fixed direction, chosen arbitrarily to be [111], and the family of orthogonal directions taken in $10^{\circ}$ intervals. This biaxial loading configuration is the elementary basis for pressurized cylinders, e.g. pipes, and is thus useful to design components based on this geometry. As well, it can serve as the design premise for loaded plates under plane stress conditions. It is of interest to note that yield surfaces can also serve as the plastic potential in the fundamental theory of plasticity (Lubliner, 2008). This equivalence is valid when the critical resolved shear stress is not dependent on the current stress state 9 (Lubliner, 2008; Starovoitov \& Naghiyev, 2012). However, this may not be applicable in the present model, where the CRSS is seen to display a strong dependence on hydrostatic (nonglide) stress components as discussed in Section 2.3.2. This is also the case in rock and soil plasticity (e.g. Pariseau et al. (1968)). In such cases, the normality rule is referred to the pressure-dependent yield surface instead.

A standing limitation of our model is that we have only made use of the tensile region of the dependence of the critical stress $\tau_{c}^{\chi}$ with the nonglide stress $\sigma$ (cf. eq. (21)). Of course, this dependence is essential to characterize the tension/compression asymmetry customarily observed in bcc crystals, cf. Section 1 . However, this is only a weak limitation, as the present CP formulation is sufficiently flexible to admit a full (nonlinear) fit to the data shown in Fig. 4. Finally, we emphasize that the present study focuses on plastic yielding, and consequently, we have not explored the evolution of the flow stress much beyond the extent needed to define a robust yield criterion (cf. Section 3.1). However, this does not detract from the validity of the dislocation density evolution model presented in Section 2.4, which has been used prolifically in many CP studies (cf. Section 1), and which is being investigated in ongoing studies.

\section{Acknowledgments}

This work was performed under the auspices of the U.S. Department of Energy by Lawrence Livermore National Laboratory under Contract No. DE-AC52-07NA27344. J. M. acknowledges support from DOE's Early Career Research Program. D. C. acknowledges support from the Consejo Social and the PhD program of the Universidad Politécnica de Madrid. The authors are indebted to Dr. M. Victoria for inspiration, encouragement, and guidance throughout this work.

\footnotetext{
${ }^{9}$ Particularly on non-deviatoric components.
} 


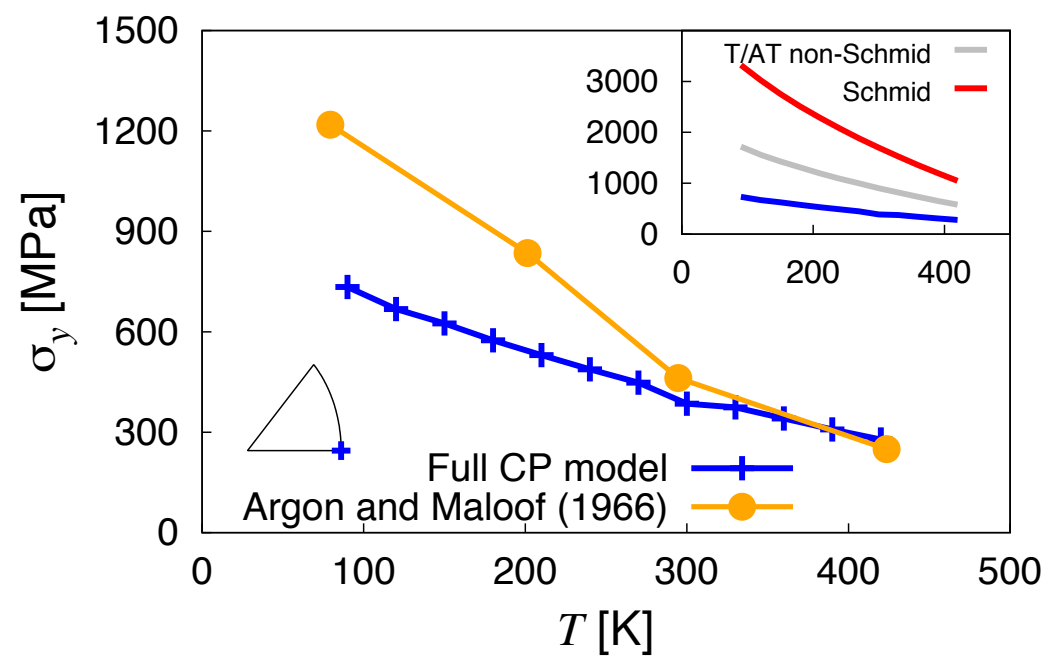

(b) [110] loading

Figure 7: Yield strength of W single crystals at the conditions used by Argon \& Maloof (1966) in tensile deformation tests under two different loading orientations. The experimental data is shown for comparison. The inset shows the results of CP calculations with different contributions of the projection tensor activated. 


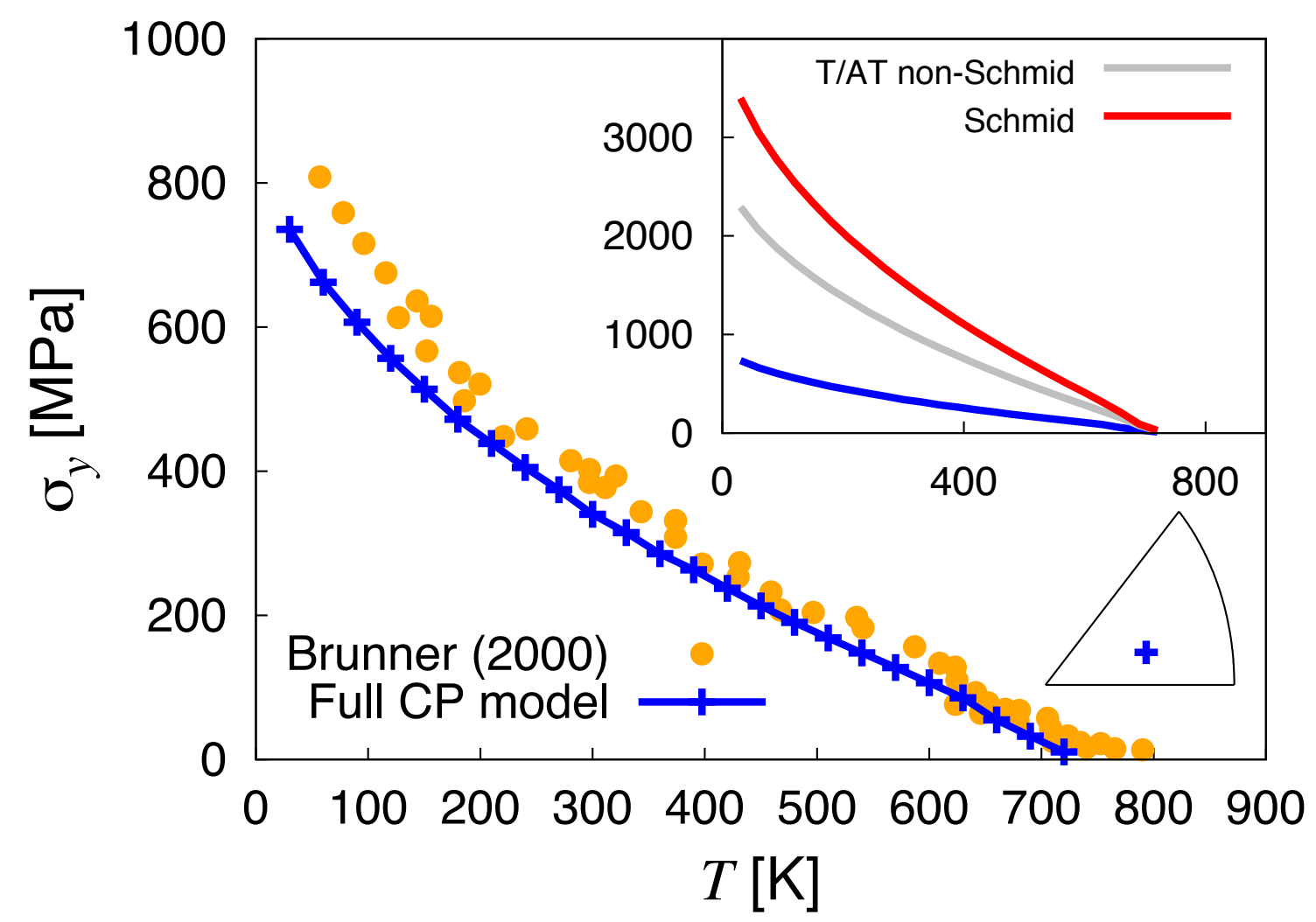

Figure 8: Yield strength of W single crystals under the conditions used by Brunner (2010) in uniaxial tensile tests. The experimental data is shown for comparison. The inset shows the results of CP calculations with different contributions of the projection tensor activated. 


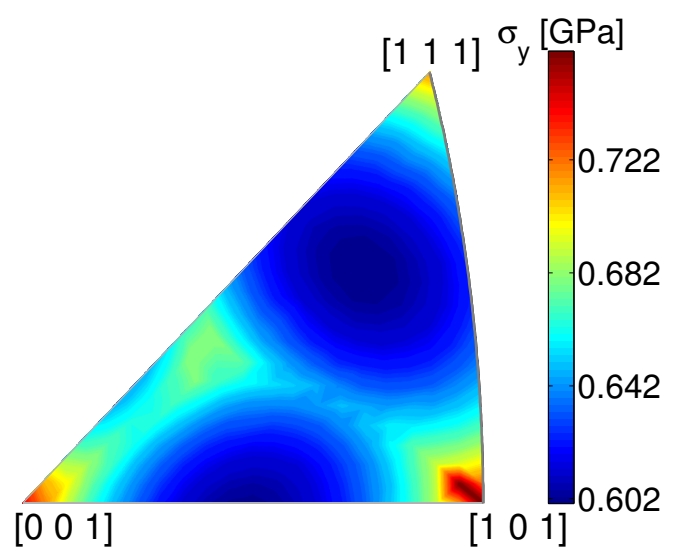

(a) $100 \mathrm{~K}$

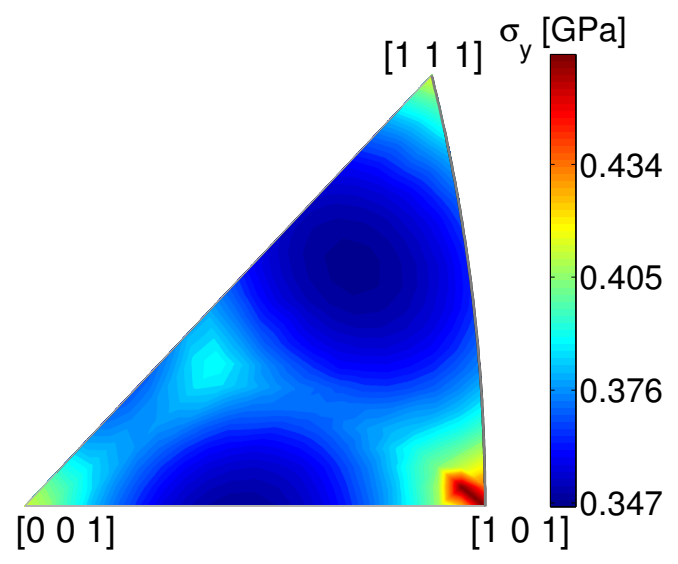

(c) $300 \mathrm{~K}$

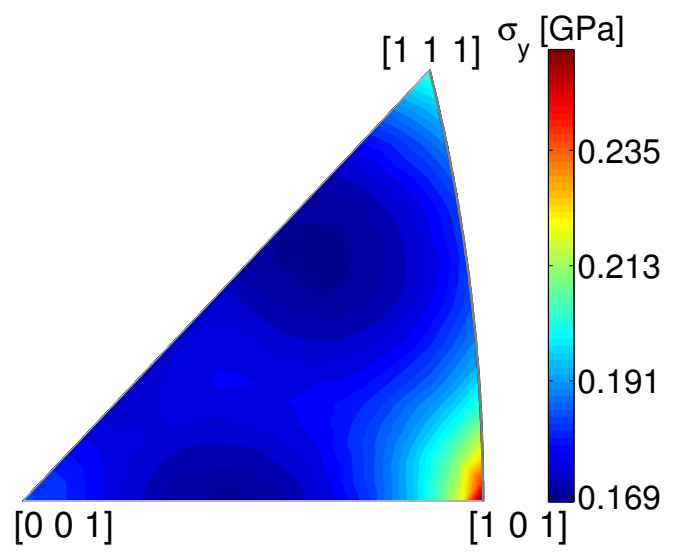

(e) $500 \mathrm{~K}$

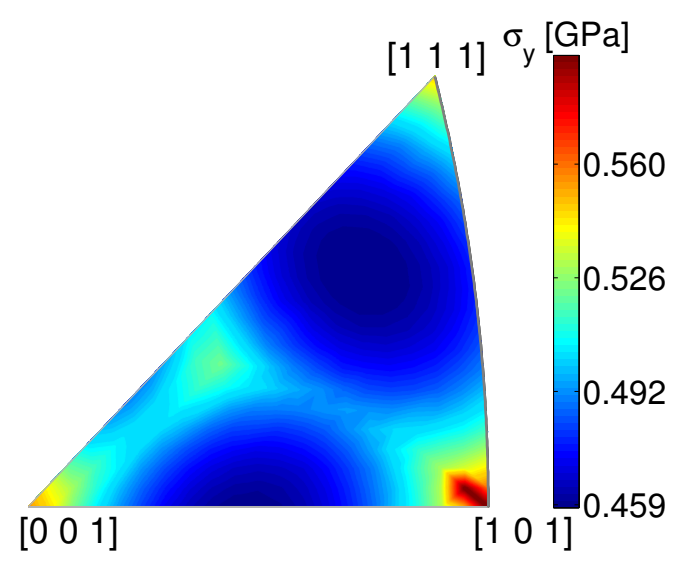

(b) $200 \mathrm{~K}$

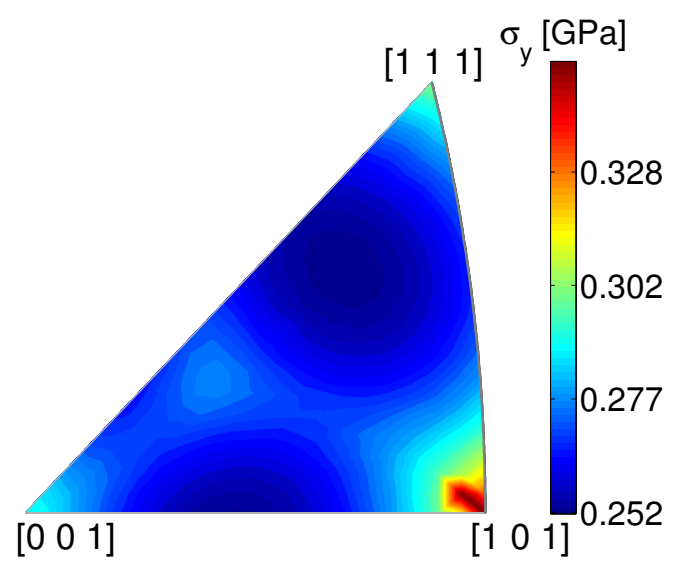

(d) $400 \mathrm{~K}$

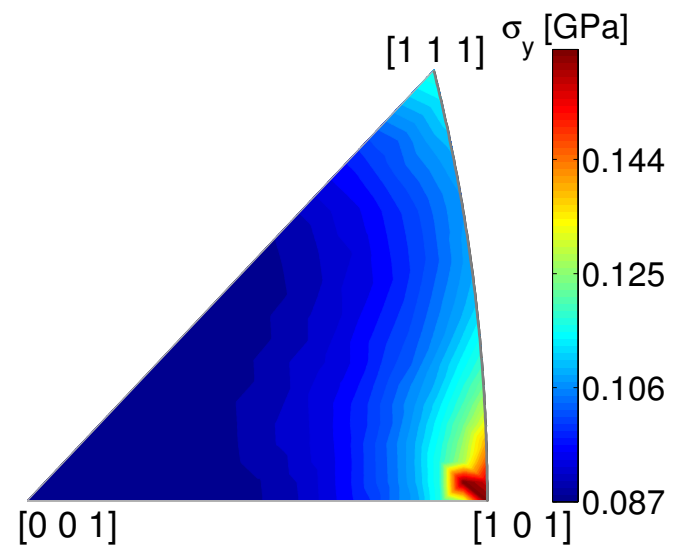

(f) $600 \mathrm{~K}$

Figure 9: Contour maps of the yield strength from uniaxial tensile test simulations for 231 uniformly distributed crystallographic orientations in the standard triangle at different temperatures. Note that each map has its own distinct numerical scale to aid in the visualization of hard and soft regions. 


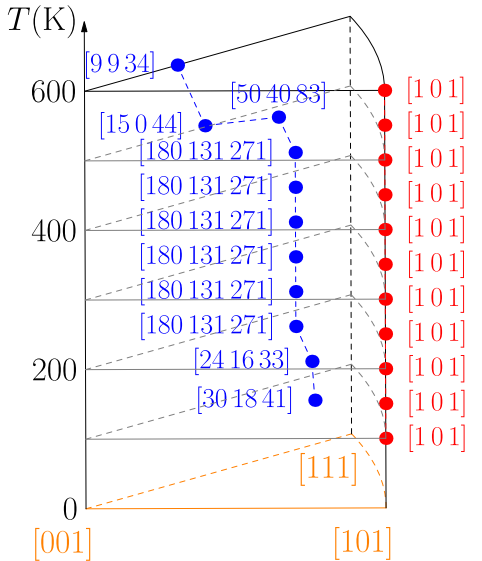

(a) $\dot{\varepsilon}=10^{-3} \mathrm{~s}^{-1}$

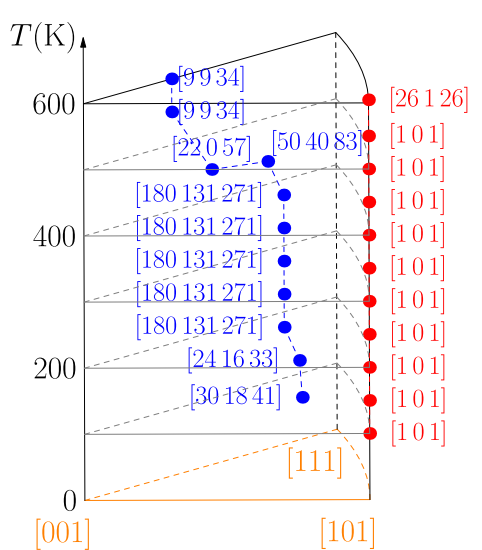

(b) $\dot{\varepsilon}=10^{-4} \mathrm{~s}^{-1}$

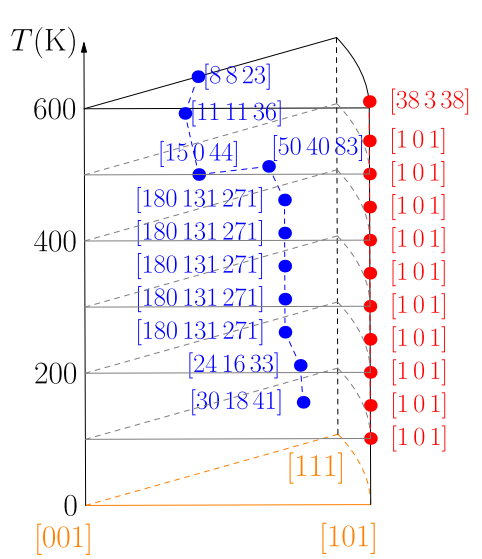

(c) $\dot{\varepsilon}=10^{-5} \mathrm{~s}^{-1}$

Figure 10: Temperature path of the softest and hardest yield directions on the standard triangle as a function of strain rate.

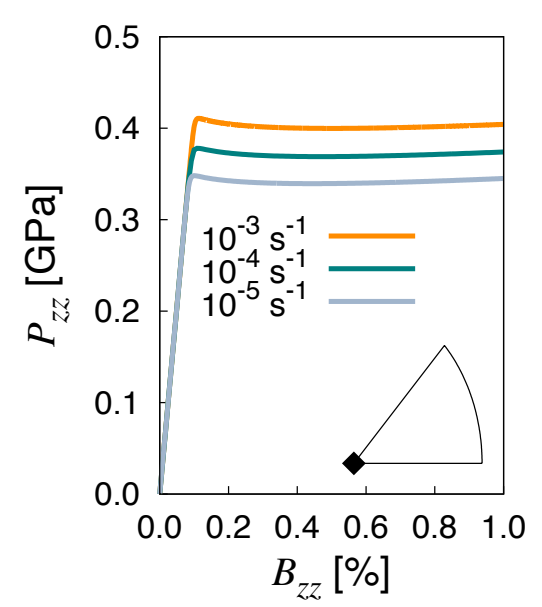

(a)

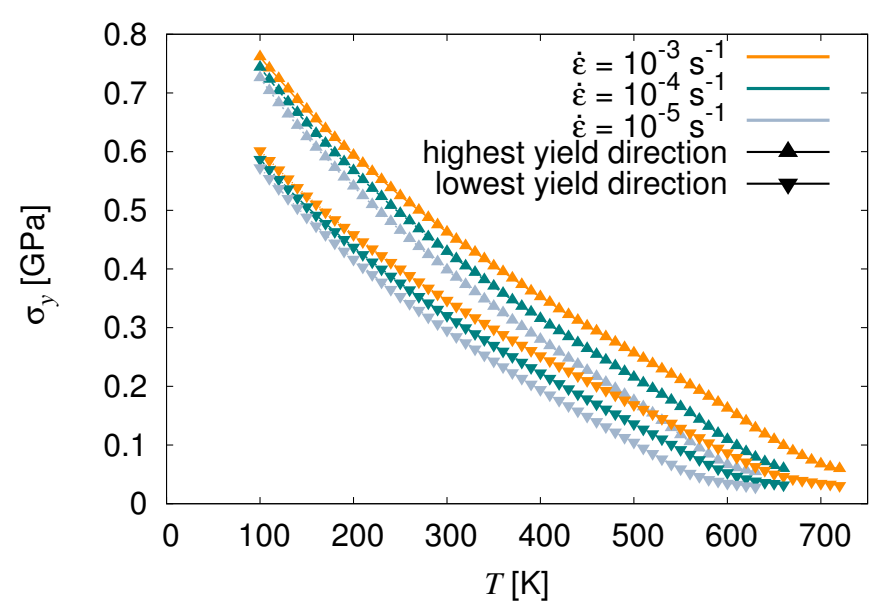

(b)

Figure 11: (a) Stress-strain relations at three different strain rates and $T=300 \mathrm{~K}$ for a [001] loading orientation. (b) Temperature dependence of the yield strength for the softest and hardest directions as a function of strain rate. 
Figure 12: Dependence of yield strength with strain rate for loading along direction [101] as a function of temperature. The inset represents the dependence of the strain rate sensitivity exponent $m$ with temperature.

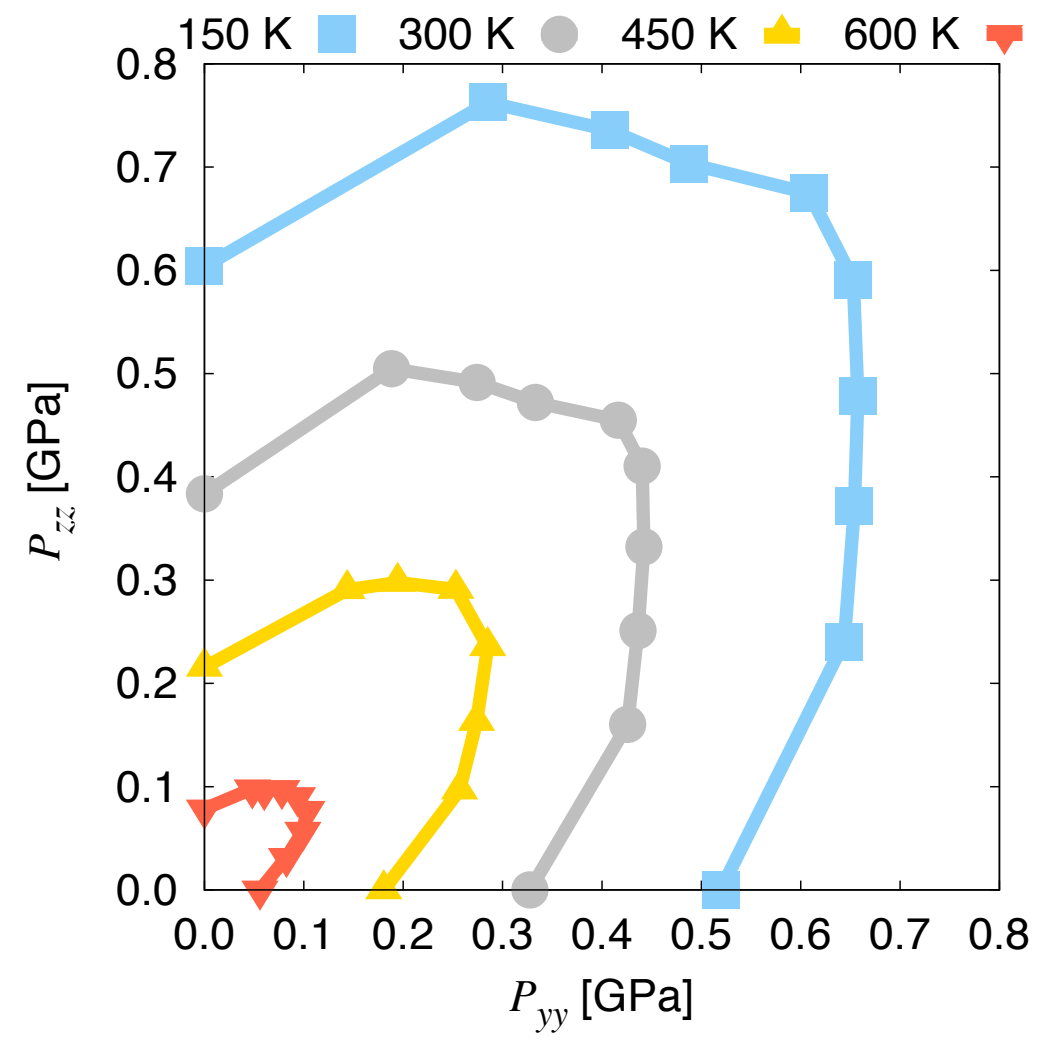

Figure 13: Yield curve for loading along directions $l_{y}=[111]$ and $l_{z}=[11 \overline{2}]$ as a function of temperature. 
Figure 14: Yield surface at $300 \mathrm{~K}$ for biaxial loading along directions belonging to the [111] zone. By symmetry, only the $60^{\circ}$-arc need be explored.

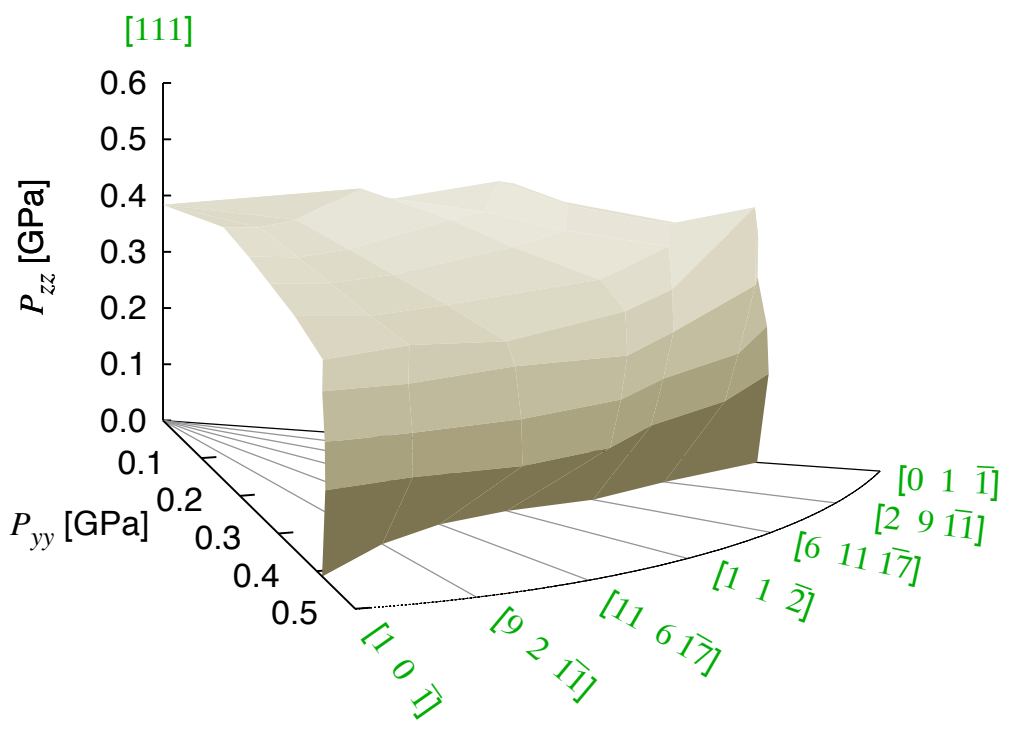


Appendix A. $\{110\}\langle 111\rangle$ slip systems and latent hardening matrix considered for bcc W.

\begin{abstract}
Table A.3: Slip systems considered in our calculations, listing the non-normalized crystallographic vectors $\boldsymbol{m}^{\alpha}, \boldsymbol{n}^{\alpha}$ and $\boldsymbol{n}_{1}^{\alpha}$. Note that in DAMASK each slip system is taken both in its positive and negative sense, which is equivalent to formulations where 24 positive slip systems are employed (Gröger et al., 2008b).
\end{abstract}

\begin{tabular}{ccccc}
\hline$\alpha$ & Reference system & $\boldsymbol{m}^{\alpha}$ & $\boldsymbol{n}^{\alpha}$ & $\boldsymbol{n}_{1}^{\alpha}$ \\
\hline 1 & {$[1 \overline{1} 1](011)$} & {$[\overline{1} \overline{1} 1]$} & {$[011]$} & {$[\overline{1} 01]$} \\
2 & {$[\overline{1} \overline{1} 1](011)$} & {$[\overline{1} \overline{1} 1]$} & {$[011]$} & {$[\overline{1} \overline{1} 0]$} \\
3 & {$[111](0 \overline{1} 1)$} & {$[\overline{1} 11]$} & {$[0 \overline{1} 1]$} & {$[1 \overline{1} 0]$} \\
4 & {$[\overline{1} 11](0 \overline{1} 1)$} & {$[\overline{1} 11]$} & {$[0 \overline{1} 1]$} & {$[101]$} \\
5 & {$[\overline{1} 11](101)$} & {$[\overline{1} 11]$} & {$[101]$} & {$[110]$} \\
6 & {$[\overline{1} \overline{1} 1](101)$} & {$[\overline{1} \overline{1} 1]$} & {$[101]$} & {$[011]$} \\
7 & {$[111](\overline{1} 01)$} & {$[111]$} & {$[\overline{1} 01]$} & {$[0 \overline{1} 1]$} \\
8 & {$[1 \overline{1} 1](\overline{1} 01)$} & {$[1 \overline{1} 1]$} & {$[\overline{1} 01]$} & {$[\overline{1} \overline{1} 0]$} \\
9 & {$[\overline{1} 11](110)$} & {$[\overline{1} 11]$} & {$[110]$} & {$[01 \overline{1}]$} \\
10 & {$[\overline{1} 1 \overline{1}](110)$} & {$[\overline{1} 1 \overline{1}]$} & {$[110]$} & {$[10 \overline{1}]$} \\
11 & {$[111](\overline{1} 10)$} & {$[111]$} & {$[\overline{1} 10]$} & {$[\overline{1} 01]$} \\
12 & {$[11 \overline{1}](\overline{1} 10)$} & {$[11 \overline{1}]$} & {$[\overline{1} 10]$} & {$[011]$} \\
\hline
\end{tabular}

Table A.4: Interaction coefficients $\xi_{\alpha \alpha^{\prime}}$ for the 12 slip systems defined in Table A.3. The letter coding employed is 'A': self; 'CP': coplanar; 'CL': collinear; 'O': orthogonal; 'G': glissile; 'S': sessile. The reader is referred to Table 1 for the numerical value of each coefficient.

\begin{tabular}{|c|cccccccccccc|}
\hline$\alpha$ & 1 & 2 & 3 & 4 & 5 & 6 & 7 & 8 & 9 & 10 & 11 & 12 \\
\hline 1 & A & & & & & & & & & & & \\
2 & CP & A & & & & & & & & & & \\
3 & S & S & A & & & & & & & & & \\
4 & S & S & CP & A & & & & & & & & \\
5 & G & O & O & CL & A & & & & & & & \\
6 & O & CL & G & O & CP & A & & & & & & \\
7 & O & G & CL & O & S & S & A & & & & & \\
8 & CL & O & O & G & S & S & CP & A & & & & \\
9 & O & G & O & CL & CL & O & G & O & A & & & \\
10 & CL & O & G & O & O & G & O & CL & CP & A & & \\
11 & G & O & CL & O & G & O & CL & O & S & S & S & \\
12 & O & CL & O & G & O & CL & O & G & S & S & CP & A \\
\hline
\end{tabular}




\section{Appendix B. Details on the atomistic calculations of non-Schmid parameters}

Critical stresses are computed by applying shear stresses incrementally to a simulation box containing a screw dislocation lying on a glide plane forming an angle $\chi$ with the MRSS plane. The system is schematically shown in Fig. 3. The box dimensions vary slightly with orientation, such that, for $\chi=0$, the box contains 3024 atoms and the dimensions are $21 a \times 24 b \times 1 c$, where $a, b$, and $c$ are the moduli of the bcc lattice vectors $x \equiv[\overline{1} 21], y \equiv[\overline{1} 01]$, and $z \equiv[111]$, respectively. The calculations are performed using the nudged elastic band (NEB) method (Henkelman et al., 2000) implemented in the parallel molecular dynamics code LAMMPS (Plimpton, 1995). Periodic boundary conditions are applied along the dislocation line direction $z$ while non-periodic and shrink-wrapped boundary conditions are applied along the $y$ and $x$ directions. The transition path selected for the NEB calculations is a linear trajectory along the reaction coordinate joining two consecutive Peierls valleys, where the dislocation is relaxed to equilibrium.

Three different forces are applied to different groups of atoms in the simulation box in order to calculate $\sigma_{\chi}^{c}$. These forces recreate the stress tensor (20) in the simulation box:

1. First, an external force $f_{z}$ is added to the atoms on the boundary surfaces of the simulation box perpendicular to the $y$-axis to study the T-AT asymmetry. The external force per atom is $f_{z}=\frac{\tau L_{x} L_{z}}{N_{z}}$, where $\tau$ is the desired shear stress, $N_{z}$ is the number of atoms in each nonperiodic surface along $z$ and $L_{x} L_{z}$ is the cross-sectional area of the each of the bounding surfaces along to $y$.

2. To study the contribution from nonglide stresses, an external force $f_{x}$ is added to the atoms on the boundaries of the simulation box perpendicular to the $x$-axis. The external force per atom is obtained as $f_{x}=\frac{\sigma L_{y} L_{z}}{N_{x}}$, where $\sigma$ is the applied nonglide stress, $N_{x}$ is the number of atoms in each surface and $L_{y} L_{z}$ is the cross-sectional area of the each of the surfaces along $x$.

3. Further, an external force $f_{y}$ is added to the atoms on the surfaces along the $y$ direction, additionally to the shear stress $\tau$. $f_{y}$ is defined as $f_{y}=\frac{\sigma L_{x} L_{z}}{N_{y}}$, with $N_{y}=N_{z}$ and $L_{x} L_{z}$ is the area of the each of the surfaces perpendicular to $z$.

31 intermediate replicas are used in the NEB calculations to capture the trajectory and measure the critical stress. 


\section{Appendix C. Crystal plasticity calculations of flow stress dependence with orientation and tempera- ture}

To demonstrate the performance of the model in the flow stress regime, we carry out calculations for a few selected orientations and temperatures up to $10 \%$ strain. Figure C.15 shows the stress-strain response at a strain rate of $10^{-3} \mathrm{~s}^{-1}$ as a function of temperature for the [001] loading orientation. This is an orientation conducive to multi-slip and thus the system is expected to harden in accordance with eqs. (9), (10), and (28) as the deformation progresses. The figure shows results for the full non-Schmid model. For general viscoplastic materials it is common to represent the $\sigma-\varepsilon$ relation as a power law of the type:

$$
\sigma=K \varepsilon^{n}
$$

where $K$ is a constant and $n$ is the so-called hardening exponent. Accordingly, the hardening rate can be expressed as:

$$
\frac{d \sigma}{d \varepsilon}=K n \varepsilon^{n-1}
$$

Fits of eq. (C.1) to the data in Fig. C.15 yield values of $n=0.82,0.86$, and 0.87 for $T=200,400$, and 600 K, respectively. From eq. (C.2), these numbers result in hardening rates of $\frac{d \sigma}{d \varepsilon} \approx 20 \mathrm{MPa} / \%$ in all cases.

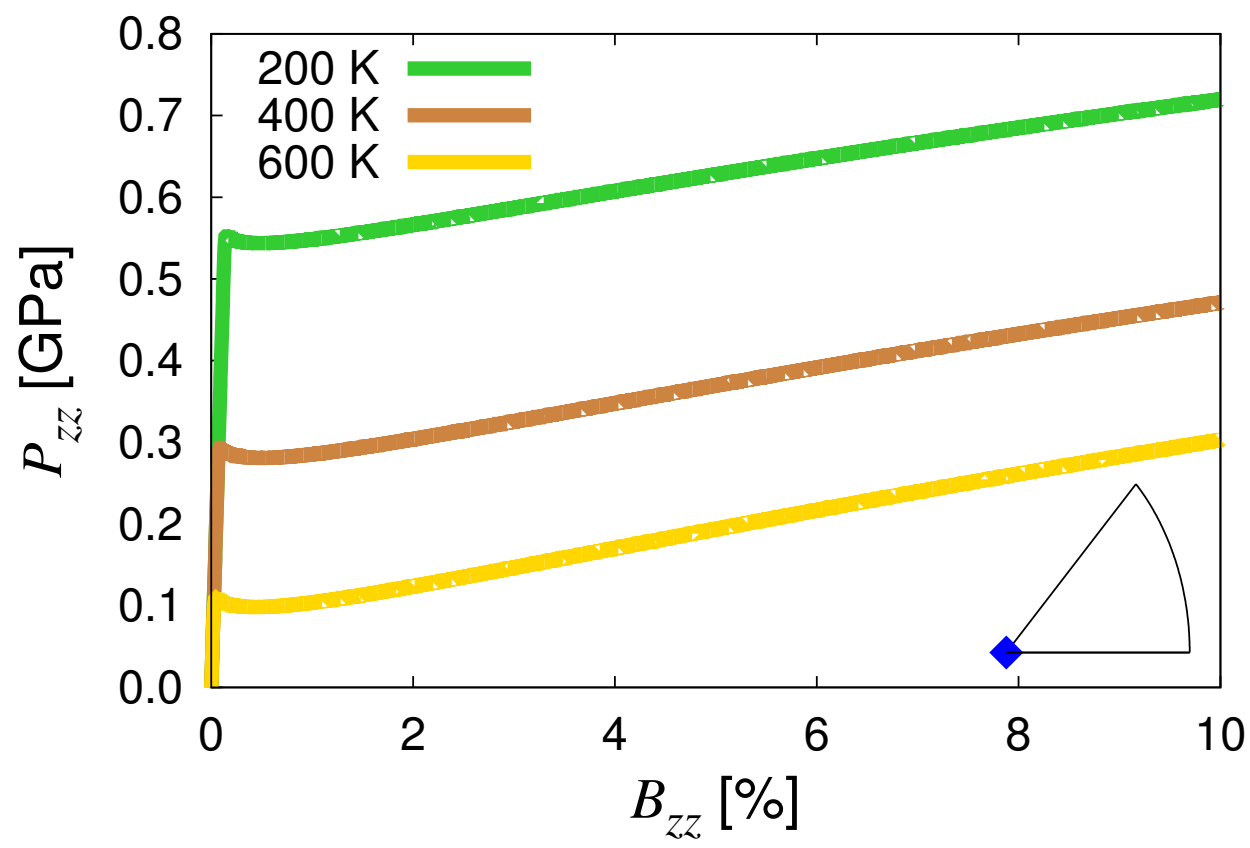

Figure C.15: Stress-strain curves for uniaxial loading along the [001] orientation at a strain rate of $10^{-3} \mathrm{~s}^{-1}$ for three different temperatures. These curves are representative of multi-slip conditions where Taylor-type hardening is enabled.

Next, we compare the model against the experimental results of Argon \& Maloof (1966) for [111] and [110] loading at $\dot{\varepsilon}=10^{-4} \mathrm{~s}^{-1}$. To avoid comparing in conditions where twinning may be operative $(<200$ K), which is not captured by our model, we carry out simulations at $293 \mathrm{~K}$. The results are shown in Figure C.16, which reveals a good agreement between the full non-Schmid model and the experimental data in the [111] loading case. According to Argon \& Maloof (1966), yielding under [110] loading occurs at approximately $460 \mathrm{MPa}$, which is immediately followed by an abrupt hardening stage that plateaus at $\varepsilon \approx$ $0.8 \%$ to a value of $\approx 760 \mathrm{MPa}$. Whether or not this is the case, this initial hardening period is not captured 
by our model. Under both loading conditions, however, the model is seen to reproduce the hardening rates in close agreement with the experimental data.

We emphasize that the results shown in Fig. C.16 have been obtained without fitting to experimental (or otherwise) stress-strain curves of any kind, and so the model appears to capture the essential features of plastic flow for the selected conditions showcased here. As noted earlier, these preliminary results do not imply that the model is suitable for calculating the flow stress under general loading conditions. 


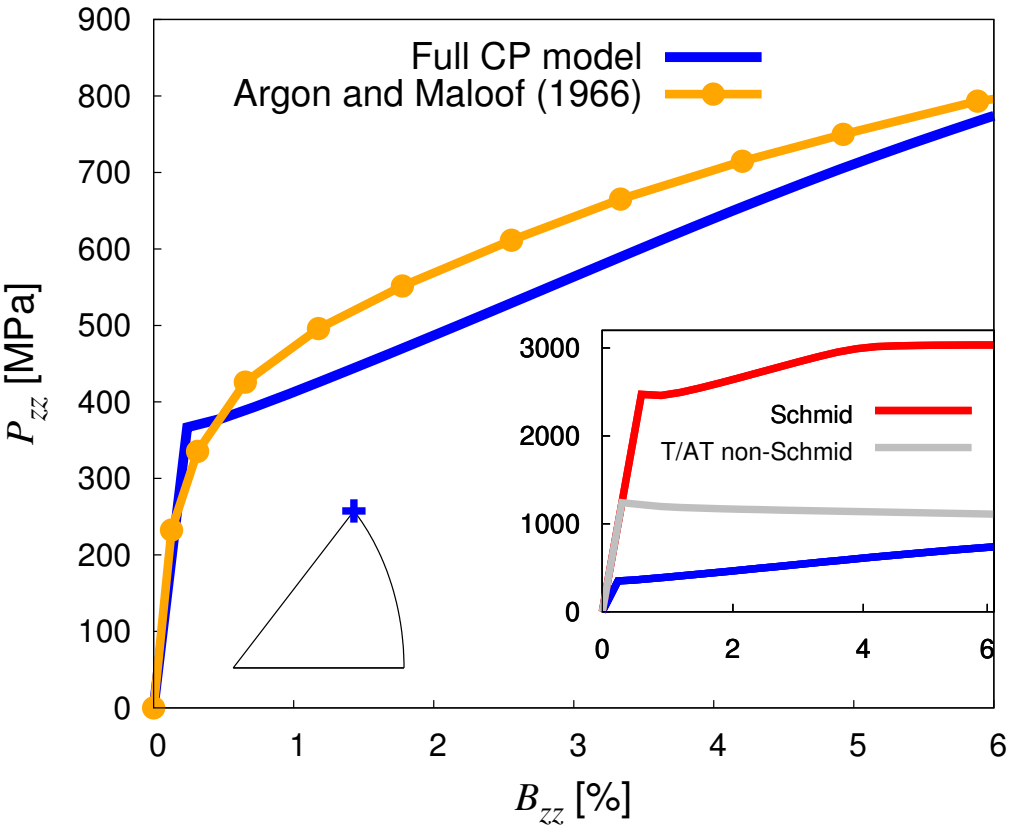

(a) [111] loading

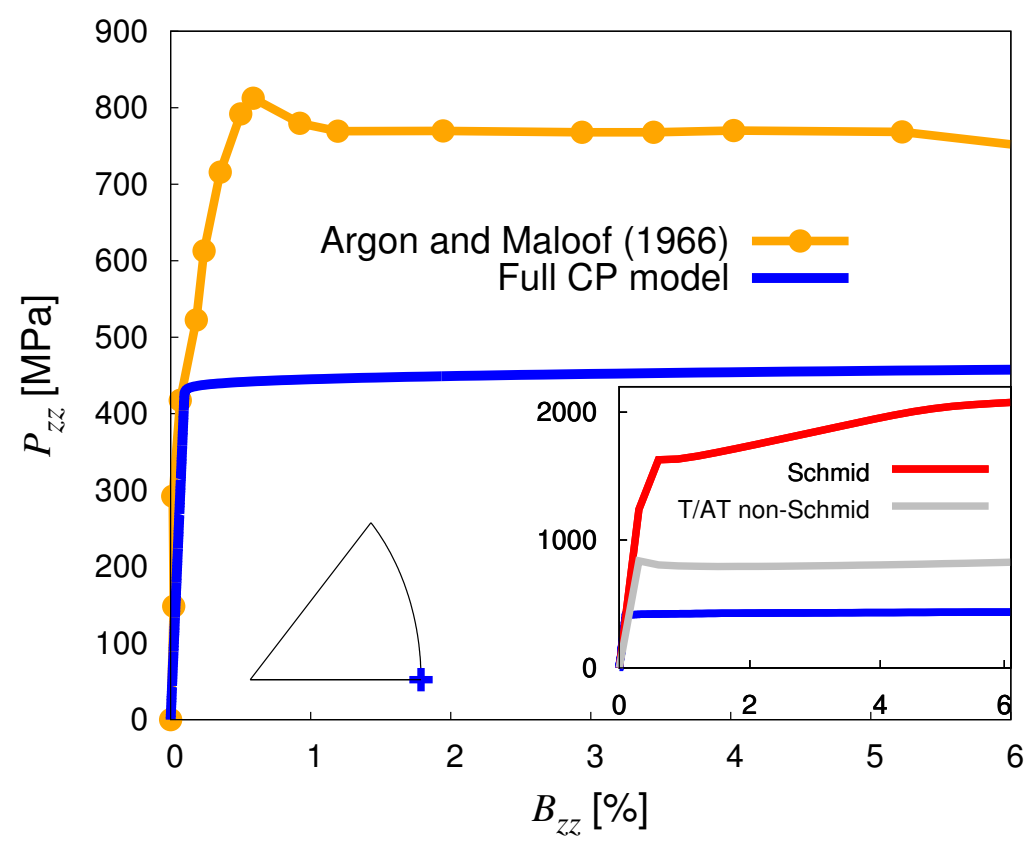

(b) [110] loading

Figure C.16: Flow stress of W single crystals at the conditions used by Argon \& Maloof (1966) (cf. Section 3.2) in tensile deformation tests under two different loading orientations. The experimental data is shown for comparison. The inset shows the results of $\mathrm{CP}$ calculations with different contributions of the projection tensor activated. 
Ackermann, F., Mughrabi, H., \& Seeger, A. (1983). Temperature-and strain-rate dependence of the flow stress of ultrapure niobium single crystals in cyclic deformation. Acta metallurgica, 31, 1353-1366. in normal direction: Analysis by an explicitly dislocation-based crystal plasticity model. International Journal of Plasticity, 52, $18-$ 32. In Honor of Hussein Zbib.

Ali, D., Mushtaq, N., \& Butt, M. (2011). Investigation of active slip-systems in some body-centered cubic metals. Journal of materials science, 46, 3812-3821.

Argon, A., \& Maloof, S. (1966). Plastic deformation of tungsten single crystals at low temperatures. Acta metallurgica, 14, 1449-1462.

Arieli, A., \& Rosen, A. (1976). Measurements of the strain rate sensitivity coefficient in superplastic ti? 6al? 4v alloy. Scripta Metallurgica, 10, 471-475.

Arsenlis, A., \& Parks, D. M. (2002). Modeling the evolution of crystallographic dislocation density in crystal plasticity. Journal of the Mechanics and Physics of Solids, 50, 1979 - 2009.

Barton, N., Bernier, J., Becker, R., Arsenlis, A., Cavallo, R., Marian, J., Rhee, M., Park, H.-S., Remington, B., \& Olson, R. (2011). A multiscale strength model for extreme loading conditions. Journal of applied physics, 109, 073501.

Basinski, S. J., \& Basinski, Z. S. (1979). Plastic deformation and work hardening. In F. R. N. Nabarro (Ed.), Dislocations in Solids (pp. 261-362). Amsterdam: North Holland volume 4.

Bodner, S. (1968). Constitutive equations for dynamic material behavior. Springer.

Bowden, P., \& Jukes, J. (1972). The plastic flow of isotropic polymers. Journal of Materials Science, 7, 52-63.

Brinckmann, S., Kim, J.-Y., \& Greer, J. R. (2008). Fundamental differences in mechanical behavior between two types of crystals at the nanoscale. Physical review letters, 100, 155502.

Brünig, M. (1997). Numerical modelling of finite elastic-plastic deformations of crystalline solids including non-schmid effects. In Computational plasticity (pp. 907-912). volume 5.

Brunner, D. (2000). Comparison of flow-stress measurements on high-purity tungsten single crystals with the kink-pair theory. Materials Transactions JIM, 41, 152-160.

Brunner, D. (2010). Temperature dependence of the plastic flow of high-purity tungsten single crystals. International Journal of Materials Research, 101, 1003-1013.

Bulatov, V., Richmond, O., \& Glazov, M. (1999). An atomistic dislocation mechanism of pressure-dependent plastic flow in aluminum. Acta materialia, 47, 3507-3514.

Bulatov, V. V., \& Cai, W. (2002). Nodal effects in dislocation mobility. Physical review letters, 89, 115501.

Cai, W., Bulatov, V. V., Justo, J. F., Argon, A. S., \& Yip, S. (2002). Kinetic monte carlo approach to modeling dislocation mobility. Computational materials science, 23, 124-130.

Caillard, D. (2010a). Kinetics of dislocations in pure fe. part i. in situ straining experiments at room temperature. Acta Materialia, 58, 3493-3503.

Caillard, D. (2010b). Kinetics of dislocations in pure fe. part ii. in situ straining experiments at low temperature. Acta Materialia, 58, 3504-3515.

Caillard, D. (2014). On the stress discrepancy at low-temperatures in pure iron. Acta Materialia, 62, 267-275.

Cereceda, D. (2015). Multiscale modeling of the plastic behaviour in single crystal tungsten: from atomistic to crystal plasticity simulations. Ph.D. thesis Univerisdad Politécnica de Madrid.

Cereceda, D., Stukowski, A., Gilbert, M., Queyreau, S., Ventelon, L., Marinica, M., Perlado, J., \& Marian, J. (2013). Assessment of interatomic potentials for atomistic analysis of static and dynamic properties of screw dislocations in w. Journal of Physics: Condensed Matter, 25, 085702.

Chang, J., Cai, W., Bulatov, V. V., \& Yip, S. (2001). Dislocation motion in bcc metals by molecular dynamics. Materials Science and Engineering: A, 309, 160-163.

Chaussidon, J., Fivel, M., \& Rodney, D. (2006). The glide of screw dislocations in bcc fe: Atomistic static and dynamic simulations. Acta materialia, 54, 3407-3416.

Chen, Z., Mrovec, M., \& Gumbsch, P. (2013). Atomistic aspects of $1 / 2\langle 111\rangle$ screw dislocation behavior in $\alpha$-iron and the derivation of microscopic yield criterion. Modelling and Simulation in Materials Science and Engineering, 21, 055023.

Christensen, R. M. (2008). Observations on the definition of yield stress. Acta Mechanica, 196, 239-244.

Christian, J. (1983). Some surprising features of the plastic deformation of body-centered cubic metals and alloys. Metallurgical Transactions A, 14, 1237-1256.

Dao, M., \& Asaro, R. (1993). Non-schmid effects and localized plastic flow in intermetallic alloys. Materials Science and Engineering: A, $170,143-160$.

Deo, C., \& Srolovitz, D. J. (2002). First passage time markov chain analysis of rare events for kinetic monte carlo: double kink nucleation during dislocation glide. Modelling and Simulation in Materials Science and Engineering, 10, 581.

Dezerald, L., Proville, L., Ventelon, L., Willaime, F., \& Rodney, D. (2015). First-principles prediction of kink-pair activation enthalpy on screw dislocations in bcc transition metals: V, nb, ta, mo, w, and fe. Physical Review B, 91, 094105.

Dezerald, L., Ventelon, L., Clouet, E., Denoual, C., Rodney, D., \& Willaime, F. (2014). Ab initio modeling of the two-dimensional energy landscape of screw dislocations in bcc transition metals. Physical Review B, 89, 024104.

Dorn, J. E., \& Rajnak, S. (1964). Nucleation of kink pairs and the peierls' ${ }^{\prime}$ mechanism of plastic deformation. Trans. Aime, 230, 1052-1064.

Drucker, D., Prager, W., \& Greenberg, H. (1952). Extended limit design theorems for continuous media. Quaterly of Applied Mathematics, 9, 381-389.

Duesbery, M. (1969). The influence of core structure on dislocation mobility. Philosophical Magazine, 19, 501-526. 
Duesbery, M., \& Foxall, R. (1969). A detailed study of the deformation of high purity niobium single crystals. Philosophical Magazine, 20, 719-751.

Duesbery, M. a.-S., \& Vitek, V. (1998). Plastic anisotropy in bcc transition metals. Acta Materialia, 46, 1481-1492.

Dumoulin, S., Hopperstad, O., \& Berstad, T. (2009). Investigation of integration algorithms for rate-dependent crystal plasticity using explicit finite element codes. Computational Materials Science, 46, 785-799.

Erieau, P., \& Rey, C. (2004). Modeling of deformation and rotation bands and of deformation induced grain boundaries in if steel aggregate during large plane strain compression. International Journal of Plasticity, 20, 1763-1788.

Escaig, B. (1968). Le Journal de Physique Colloques, 29.

Escaig, B. (1974). Dissociation and mechanical properties. dislocation splitting and the plastic glide process in crystals. Le Journal de Physique Colloques, 35, C7-151.

Estrin, Y. (1996). Dislocation-density-related constitutive modeling. Unified constitutive laws of plastic deformation?,(Eds. AS Krausz and K. Krausz), Academic Press.

Fish, J., \& Shek, K. (2000). Finite deformation plasticity based on the additive split of the rate of deformation and hyperelasticity. Computer methods in applied mechanics and engineering, 190, 75-93.

Franciosi, P. (1983). Glide mechanisms in bcc crystals: an investigation of the case of $\alpha$-iron through multislip and latent hardening tests. Acta Metallurgica, 31, 1331-1342.

Franciosi, P. (1985). The concepts of latent hardening and strain hardening in metallic single crystals. Acta Metallurgica, 33, 16011612.

Franciosi, P., Le, L., Monnet, G., Kahloun, C., \& Chavanne, M.-H. (2015). Investigation of slip system activity in iron at room temperature by $\{\mathrm{SEM}\}$ and $\{\mathrm{AFM}\}$ in-situ tensile and compression tests of iron single crystals. International Journal of Plasticity, 65, 226 - 249. URL: http: / /www. sciencedirect.com/science/article/pii/S0749641914001922. doi:http: //dx.doi .org/10. $1016 /$ j.ijplas.2014.09.008.

Gilbert, M., Queyreau, S., \& Marian, J. (2011). Stress and temperature dependence of screw dislocation mobility in $\alpha$-fe by molecular dynamics. Physical Review B, 84, 174103.

Gordon, P., Neeraj, T., Li, Y., \& Li, J. (2010). Screw dislocation mobility in bcc metals: the role of the compact core on double-kink nucleation. Modelling and Simulation in Materials Science and Engineering, 18, 085008.

Gröger, R., Bailey, A., \& Vitek, V. (2008a). Multiscale modeling of plastic deformation of molybdenum and tungsten: I. atomistic studies of the core structure and glide of $1 / 2\langle 111\rangle$ screw dislocations at 0k. Acta Materialia, 56, 5401-5411.

Gröger, R., Racherla, V., Bassani, J., \& Vitek, V. (2008b). Multiscale modeling of plastic deformation of molybdenum and tungsten: Ii. yield criterion for single crystals based on atomistic studies of glide of $1 / 2\langle 111\rangle$ screw dislocations. Acta Materialia, 56, 5412-5425. Gröger, R., \& Vitek, V. (2005). Breakdown of the schmid law in bcc molybdenum related to the effect of shear stress perpendicular to the slip direction. In Materials Science Forum (pp. 123-126). Trans Tech Publ volume 482.

Gröger, R., \& Vitek, V. (2007). Explanation of the discrepancy between the measured and atomistically calculated yield stresses in body-centred cubic metals. Philosophical magazine letters, 87, 113-120.

Gröger, R., \& Vitek, V. (2008). Multiscale modeling of plastic deformation of molybdenum and tungsten. iii. effects of temperature and plastic strain rate. Acta Materialia, 56, 5426-5439.

Gupta, I., \& Li, J. (1970). Stress relaxation, internal stress, and work hardening in some bcc metals and alloys. Metallurgical Transactions, 1, 2323-2330. URL: http: / / dx . doi.org/10.1007/BF 02643451. doi:10.1007/BF02643451.

Hamelin, C., Diak, B., \& Pilkey, A. (2011). Multiscale modelling of the induced plastic anisotropy in bcc metals. International Journal of Plasticity, 27, 1185-1202.

Hedworth, J., \& Stowell, M. (1971). The measurement of strain-rate sensitivity in superplastic alloys. Journal of Materials Science, 6, 1061-1069.

Henkelman, G., Johannesson, G., \& Jonsson, H. (2000). Methods for Finding Saddle Points and Minimum Energy Paths. Kluwer Academic Publishers.

Hölscher, M., Raabe, D., \& Lücke, K. (1991). Rolling and recrystallization textures of bcc steels. Steel Research International, (pp. 567-575).

Hölscher, M., Raabe, D., \& Lücke, K. (1994). Relationship between rolling textures and shear textures in fcc and bcc metals. Acta metallurgica et materialia, 42, 879-886.

Hull, D., Byron, J., \& Noble, F. (1967). Orientation dependence of yield in body-centered cubic metals. Canadian Journal of Physics, 45, 1091-1099.

Hutchinson, J., \& Neale, K. (1977). Influence of strain-rate sensitivity on necking under uniaxial tension. Acta Metallurgica, 25, 839-846.

Ito, K., \& Vitek, V. (2001). Atomistic study of non-schmid effects in the plastic yielding of bcc metals. Philosophical Magazine A, 81, 1387-1407.

Kalidindi, S. R., Bronkhorst, C. A., \& Anand, L. (1992). Crystallographic texture evolution in bulk deformation processing of fcc metals. J. Mech. Phys. Solids, 40, 537-569.

Kitayama, K., Tomé, C., Rauch, E., Gracio, J., \& Barlat, F. (2013). A crystallographic dislocation model for describing hardening of polycrystals during strain path changes. application to low carbon steels. International Journal of Plasticity, 46, 54-69.

Klopp, W. D., Witzke, W. R., \& Raffo, P. L. (1964). Effects of Grain Size on the Tensile and Creep Properties of Arc-Melted and ElectrobeamMelted Tungsten at $2250^{\circ}$ TO $4140^{\circ} \mathrm{F}$. Technical Report E-2681 National Aeronautics and Aerospace Administration Lewis Research Center, Cleveland, Ohio.

Knezevic, M., Beyerlein, I. J., Lovato, M. L., Tomé, C. N., Richards, A. W., \& McCabe, R. J. (2014). A strain-rate and temperature dependent constitutive model for bcc metals incorporating non-schmid effects: Application to tantalum-tungsten alloys. International Journal of Plasticity, 62, 93-104.

Kocks, U., Argon, A., \& Ashby, M. (1975). Progress in materials science. Thermodynamics and Kinetics of Slip, 19, 110-170. 
Koester, A., Ma, A., \& Hartmaier, A. (2012). Atomistically informed crystal plasticity model for body-centered cubic iron. Acta Materialia 60, 3894-3901.

Komanduri, R., Chandrasekaran, N., \& Raff, L. (2001). Molecular dynamics (md) simulation of uniaxial tension of some single-crystal cubic metals at nanolevel. International Journal of Mechanical Sciences, 43, 2237-2260.

Kuchnicki, S., Radovitzky, R., \& Cuitiño, A. (2008). An explicit formulation for multiscale modeling of bcc metals. International Journal of Plasticity, 24, 2173-2191.

Lee, E. H. (1969). Elastic-plastic deformation at finite strains. Journal of Applied Mechanics, 36, 1-6.

Lee, Y. J., Subhash, G., \& Ravichandran, G. (1999). Constitutive modeling of textured body-centered-cubic (bcc) polycrystals. International Journal of Plasticity, 15, 625-645.

Li, H., Wurster, S., Motz, C., Romaner, L., Ambrosch-Draxl, C., \& Pippan, R. (2012). Dislocation-core symmetry and slip planes in tungsten alloys: Ab initio calculations and microcantilever bending experiments. Acta Materialia, 60, 748-758.

Lim, H., Battaile, C. C., Carroll, J. D., Boyce, B. L., \& Weinberger, C. R. (2015a). A physically based model of temperature and strain rate dependent yield in bcc metals: Implementation into crystal plasticity. Journal of the Mechanics and Physics of Solids, 74, 80-96.

Lim, H., Hale, L., Zimmerman, J., Battaile, C., \& Weinberger, C. (2015b). A multi-scale model of dislocation plasticity in $\alpha$-fe: Incorporating temperature, strain rate and non-schmid effects. International Journal of Plasticity, .

Lim, H., Weinberger, C. R., Battaile, C. C., \& Buchheit, T. E. (2013). Application of generalized non-schmid yield law to low-temperature plasticity in bcc transition metals. Modelling and Simulation in Materials Science and Engineering, 21, 045015.

Lin, K., \& Chrzan, D. (1999). Kinetic monte carlo simulation of dislocation dynamics. Physical Review B, 60, 3799.

Lubliner, J. (2008). Plasticity theory. Courier Corporation.

Ma, A., Roters, F., \& Raabe, D. (2007). A dislocation density based constitutive law for $\{\mathrm{BCC}\}$ materials in crystal plasticity $\{\mathrm{FEM}\}$. Computational Materials Science, 39, 91 - 95. Proceedings of the 15th International Workshop on Computational Mechanics of Materials The 15th International Workshop on Computational Mechanics of Materials.

Madec, R., \& Kubin, L. (2004). Dislocation interactions and symmetries in bcc crystals. In IUTAM Symposium on Mesoscopic Dynamics of Fracture Process and Materials Strength (pp. 69-78). Springer.

Marichal, C., Srivastava, K., Weygand, D., Van Petegem, S., Grolimund, D., Gumbsch, P., \& Van Swygenhoven, H. (2014). Origin of anomalous slip in tungsten. Phys. Rev. Lett., 113, 025501. URL: ht tp / / / ink. aps. org/doi / 10.1103/PhysRevLett . 113.025501. doi:10.1103/PhysRevLett.113.025501.

Marichal, C., Van Swygenhoven, H., Van Petegem, S., \& Borca, C. (2013). $\{110\}$ slip with $\{112\}$ slip traces in bcc tungsten. Scientific reports, 3, 2547.

McDowell, D. L. (2008). Viscoplasticity of heterogeneous metallic materials. Materials Science and Engineering: R: Reports, 62, 67-123. Mecking, H., \& Kocks, U. (1981). Kinetics of flow and strain-hardening. Acta Metallurgica, 29, 1865-1875.

Monnet, G., Domain, C., Queyreau, S., Naamane, S., \& Devincre, B. (2009). Atomic and dislocation dynamics simulations of plastic deformation in reactor pressure vessel steel. Journal of Nuclear Materials, 394, 174 - 181.

Monnet, G., Naamane, S., \& Devincre, B. (2011). Orowan strengthening at low temperatures in bcc materials studied by dislocation dynamics simulations. Acta Materialia, 59, $451-461$.

Naamane, S., Monnet, G., \& Devincre, B. (2010). Low temperature deformation in iron studied with dislocation dynamics simulations. International Journal of Plasticity, 26, $84-92$.

Narayanan, S., McDowell, D. L., \& Zhu, T. (2014). Crystal plasticity model for $\{B C C\}$ iron atomistically informed by kinetics of correlated kinkpair nucleation on screw dislocation. Journal of the Mechanics and Physics of Solids, 65, 54 - 68.

Pariseau, W. G. et al. (1968). Plasticity theory for anisotropic rocks and soil. In The 10th US Symposium on Rock Mechanics (USRMS). American Rock Mechanics Association.

Patra, A., Zhu, T., \& McDowell, D. L. (2014). Constitutive equations for modeling non-schmid effects in single crystal bcc-fe at low and ambient temperatures. International Journal of Plasticity, 59, $1-14$.

Peeters, B., Kalidindi, S., Van Houtte, P., \& Aernoudt, E. (2000). A crystal plasticity based work-hardening/softening model for bcc metals under changing strain paths. Acta materialia, 48, 2123-2133.

Pichl, W. (2002). Slip geometry and plastic anisotropy of body-centered cubic metals. physica status solidi (a), 189, 5-25.

Plimpton, S. (1995). Fast Parallel Algorithms For Short-Range Molecular-Dynamics. http://lammps.sandia.gov. Journal Of Computational Physics, 117, 1-19.

Prager, W. (1952). The general theory of limit design. In Proceedings of the 8th International Congress on theoretical and Applied Mechanics, Istanbul (pp. 65-72). volume 19.

Proville, L., Rodney, D., \& Marinica, M.-C. (2012). Quantum effect on thermally activated glide of dislocations. Nature materials, 11, 845-849.

Qin, Q., \& Bassani, J. L. (1992). Non-schmid yield behavior in single crystals. Journal of the Mechanics and Physics of Solids, 40, 813 833.

Queyreau, S., Marian, J., Gilbert, M., \& Wirth, B. (2011). Edge dislocation mobilities in bcc fe obtained by molecular dynamics. Physical Review B, 84, 064106.

Queyreau, S., Monnet, G., \& Devincre, B. (2009). Slip systems interactions in $\alpha$-iron determined by dislocation dynamics simulations. International Journal of Plasticity, 25, $361-377$.

Raabe, D. (1995a). Investigation of contribution of $\{123\}$ slip planes to development of rolling textures in bee metals by use of taylor models. Materials science and technology, 11, 455-460.

Raabe, D. (1995b). Simulation of rolling textures of bcc metals considering grain interactions and crystallographic slip on $\{110\},\{112\}$ and $\{123\}$ planes. Materials Science and Engineering: A, 197, 31-37.

Raabe, D., Schlenkert, G., Weisshaupt, H., \& Lücke, K. (1994). Texture and microstructure of rolled and annealed tantalum. Materials science and technology, 10, 299-305.

Raffo, P. L. (1969). Yielding and fracture in tungsten and tungsten-rhenium alloys. Journal of the Less Common Metals, 17, $133-149$. 
Raphanel, J., \& Van Houtte, P. (1985). Simulation of the rolling textures of bcc metals by means of the relaxed taylor theory. Acta Metallurgica, 33, 1481-1488.

Reina, C., \& Conti, S. (2014). Kinematic description of crystal plasticity in the finite kinematic framework: A micromechanical understanding of F=FeFp. Journal of the Mechanics and Physics of Solids, 67, $40-61$.

Romaner, L., Ambrosch-Draxl, C., \& Pippan, R. (2010). Effect of rhenium on the dislocation core structure in tungsten. Physical review letters, 104, 195503.

Roters, F. (2011). Advanced material models for the crystal plasticity finite element method: development of a general CPFEM framework. Universitätsbibliothek.

Roters, F., Eisenlohr, P., Hantcherli, L., Tjahjanto, D., Bieler, T., \& Raabe, D. (2010). Overview of constitutive laws, kinematics, homogenization and multiscale methods in crystal plasticity finite-element modeling: Theory, experiments, applications. Acta Materialia, $58,1152-1211$.

Roters, F., Eisenlohr, P., Kords, C., Tjahjanto, D., Diehl, M., \& Raabe, D. (2012). DAMASK: the Dusseldorf Advanced MAterial Simulation Kit for studying crystal plasticity using an FE based or a spectral numerical solver. Procedia IUTAM, 3, 3 - 10. IUTAM Symposium on Linking Scales in Computations: From Microstructure to Macro-scale Properties.

Samolyuk, G. D., Osetsky, Y., \& Stoller, R. (2013). The influence of transition metal solutes on the dislocation core structure and values of the peierls stress and barrier in tungsten. Journal of Physics: Condensed Matter, 25, 025403.

Scarle, S., Ewels, C., Heggie, M., \& Martsinovich, N. (2004). Linewise kinetic monte carlo study of silicon dislocation dynamics. Physical Review $B, 69,075209$.

Schmid, E., \& Boas, W. (1935). Kristallplastizität: mit besonderer Berücksichtigung der Metalle volume 17. J. Springer

Seeger, A. (1981). The temperature and strain-rate dependence of the flow stress of body-centred cubic metals: A theory based on kink-kink interactions. Zeitschrift fur Metallkunde, 72, 369-380.

Seeger, A. (1995). The flow stress of high-purity refractory body-centred cubic metals and its modification by atomic defects. Le Journal de Physique IV, 5, C7-45.

Serenelli, M., Bertinetti, M., \& Signorelli, J. (2010). Investigation of the dislocation slip assumption on formability of $\{$ BCC $\}$ sheet metals. International Journal of Mechanical Sciences, 52, 1723 - 1734.

Šesták, B., \& Zárubová, N. (1965). Asymmetry of slip in fe-si alloy single crystals. physica status solidi (b), 10, 239-250.

Sheng, D., Sloan, S., \& Gens, A. (2004). A constitutive model for unsaturated soils: thermomechanical and computational aspects. Computational Mechanics, 33, 453-465.

Sherwood, P., Guiu, F., Kim, H.-C., \& Pratt, P. L. (1967). Plastic anisotropy of tantalum, niobium, and molybdenum. Canadian Journal of Physics, 45, 1075-1089.

Soare, S. C. (2014). Plasticity and non-schmid effects. Proceedings of the Royal Society A: Mathematical, Physical and Engineering Science, 470, 20130440.

Stainier, L., Cuitiño, A. M., \& Ortiz, M. (2002). A micromechanical model of hardening, rate sensitivity and thermal softening in bcc single crystals. Journal of the Mechanics and Physics of Solids, 50, 1511-1545.

Starovoitov, E., \& Naghiyev, F. B. O. (2012). Foundations of the Theory of Elasticity, Plasticity, and Viscoelasticity. CRC Press.

Stephens, J. R. (1970). Dislocation structures in single-crystal tungsten and tungsten alloys. Metallurgical and Materials Transactions, 1, 1293-1301.

Stukowski, A., Cereceda, D., Swinburne, T. D., \& Marian, J. (2015). Thermally-activated non-schmid glide of screw dislocations in w using atomistically-informed kinetic monte carlo simulations. International Journal of Plasticity, 65, 108-130.

Swinburne, T., Dudarev, S., Fitzgerald, S., Gilbert, M., \& Sutton, A. (2013). Theory and simulation of the diffusion of kinks on dislocations in bcc metals. Physical Review B, 87, 064108.

Takeuchi, S., Furubayashi, E., \& Taoka, T. (1967). Orientation dependence of yield stress in 4.4\% silicon iron single crystals. Acta metallurgica, 15, 1179-1191.

Tang, M., \& Marian, J. (2014). Temperature and high strain rate dependence of tensile deformation behavior in single-crystal iron from dislocation dynamics simulations. Acta Materialia, 70, 123-129.

Taylor, G. (1928). The deformation of crystals of $\beta$-brass. Proceedings of the Royal Society of London. Series A, 118, 1-24.

Taylor, G. (1934a). The mechanism of plastic deformation of crystals. part ii. comparison with observations. Proceedings of the Royal Society of London. Series A, Containing Papers of a Mathematical and Physical Character, (pp. 388-404).

Taylor, G. (1992). Thermally-activated deformation of bcc metals and alloys. Progress in materials science, 36, $29-61$.

Taylor, G. I. (1934b). The mechanism of plastic deformation of crystals. part i. theoretical. Proceedings of the Royal Society of London. Series A, Containing Papers of a Mathematical and Physical Character, (pp. 362-387).

Ventelon, L., \& Willaime, F. (2007). Core structure and peierls potential of screw dislocations in $\alpha$-fe from first principles: cluster versus dipole approaches. Journal of Computer-Aided Materials Design, 14, 85-94.

Ventelon, L., Willaime, F., Clouet, E., \& Rodney, D. (2013). Ab initio investigation of the peierls potential of screw dislocations in bcc fe and w. Acta Materialia, 61, 3973-3985.

Vitek, V. (2004). Core structure of screw dislocations in body-centred cubic metals: relation to symmetry and interatomic bonding. Philosophical Magazine, 84, 415-428.

Vitek, V., \& Yamaguchi, M. (1973). Core structure of nonscrew 1/2 (111) dislocations on (110) planes in bcc crystals. ii. peierls stress and the effect of an external shear stress on the cores. Journal of Physics F: Metal Physics, 3, 537.

Weinberger, C. R., Battaile, C. C., Buchheit, T. E., \& Holm, E. A. (2012). Incorporating atomistic data of lattice friction into bcc crystal plasticity models. International Journal of Plasticity, 37, 16-30.

Weinberger, C. R., Tucker, G. J., \& Foiles, S. M. (2013). Peierls potential of screw dislocations in bcc transition metals: Predictions from density functional theory. Physical Review B, 87, 054114.

Woodward, C., \& Rao, S. (2001). Ab-initio simulation of isolated screw dislocations in bcc mo and ta. Philosophical Magazine A, 81, 1305-1316. 
Wurster, S., Gludovatz, B., \& Pippan, R. (2010). High temperature fracture experiments on tungsten-rhenium alloys. International Journal of Refractory Metals and Hard Materials, 28, 692-697.

64 Yalcinkaya, T., Brekelmans, W., \& Geers, M. (2008). Bcc single crystal plasticity modeling and its experimental identification. Modelling and Simulation in Materials Science and Engineering, 16, 085007.

6 Yang, L., Söderlind, P., \& Moriarty, J. A. (2001). Accurate atomistic simulation of $(\mathrm{a} / 2)<111>$ screw dislocations and other defects in bcc tantalum. Philosophical Magazine A, 81, 1355-1385.

868 Zurek, A. K., \& Gray III, G. T. (1991). Dynamic strength and strain rate effects on fracture behavior of tungsten and tungsten alloys. 869 Journal de Physique, 1, 631-637.

870 Zwiesele, S., \& Diehl, J. (1979). Temperature and strain rate dependence of the macro-yield stress of high-purity iron single crystals. 871 Strength of Metals and Alloys, 1, 59-64. 